Comparative Population Studies

Selected Articles in German Vol. 40 (2015): 3-32

(Erstveröffentlichung: 05.12.2014)

\title{
Ost-West-Paare: Verbreitung, Eigenschaften und Stabilität
}

\section{Daniel Lois}

Zusammenfassung: Auf der Basis von SOEP-Daten werden Partnerschaften untersucht, die jeweils aus einem westdeutsch und ostdeutsch sozialisierten Partner bestehen und aktuell in den alten oder neuen Bundesländern wohnen. Der geschätzte Anteil der Ost-West-Paare an allen Ehen bzw. nichtehelichen Lebensgemeinschaften erhöht sich im Beobachtungszeitraum kontinuierlich und liegt im Jahr 2009 bei etwa zwei bzw. elf Prozent. Die speziellen Charakteristika der Ost-West-Paare bestehen darin, dass die außerhäusliche Arbeitsteilung relativ egalitär ausgerichtet ist, die Partner sich überdurchschnittlich oft in ihrer konfessionellen Zugehörigkeit sowie religiösen Praxis unterscheiden, Scheidungserfahrungen mindestens eines Partners häufig vorkommen und auch eine starke Neigung zur Nichtehelichkeit festzustellen ist. Neben dem Ort der Sozialisation kommt dem aktuellen Wohnort im Hinblick auf die ökonomische Lage, die Arbeitsteilung und die Heiratsneigung eine eigenständige Bedeutung zu. Analysen zur Partnerschaftsstabilität zeigen, dass Ost-West-Paare ein relativ hohes Trennungsrisiko aufweisen. Dies ist teilweise auf religiöse Differenzen zwischen den Partnern, vor allem aber auf die geringe Heiratsneigung und die Überrepräsentation bereits geschiedener Personen in diesem Partnerschaftstyp zurückzuführen.

Schlagwörter: Ost-West-Unterschiede · Binnenmigration · Partnerschaftsstabilität • Homogamie

\section{$1 \quad$ Einleitung}

Unmittelbar nach der Wiedervereinigung vor mehr als zwei Jahrzehnten haben erhebliche innerdeutsche Migrationsströme eingesetzt. Nach Fuchs-Schündeln/ Schündeln (2009: 32-35) sind, inklusive der Rückkehrer und Mehrfachmobilen, zwischen 1991 und 2006 etwa zweieinhalb Millionen Menschen von den neuen in die alten Bundesländer gewandert und etwa eine Million von den alten in die neuen Bundesländer. Verheiratete Personen sind innerhalb der Population der Binnenmigranten deutlich unterrepräsentiert (Fuchs-Schündeln/Schündeln 2009). Daher 
ist zu erwarten, dass mittlerweile zahlreiche Ost-West-Paare entstanden sind, z.B. bestehend aus einer in der ehemaligen DDR sozialisierten Frau und einem in der alten BRD aufgewachsenen Mann. Das Phänomen der Ost-West-Liebe scheint eine gewisse Faszination auszuüben, was sich allein schon an einigen medienwirksamen Aktionen ablesen lässt: So trafen sich am 9. Januar 2009 über 100 Ost-West-Paare in Berlin-Mitte, um mit Wolfgang Tiefensee, dem damaligen Beauftragten der Bundesregierung für die neuen Bundesländer, über den Stand der Wiedervereinigung zu sprechen (Berliner Kurier, 10.01.2009) und im Februar 2013 suchte Udo Lindenberg zehn Ost-West-Liebespärchen für sein Musical "Hinterm Horizont" (Hamburger Abendblatt, 19.02.13)

Inwieweit die Ost-West-Dimension für Partnerwahlprozesse relevant ist, wird durch die bisherige Forschung nicht eindeutig beantwortet. Eine Studie von Rösler (2010) zeigt, dass einige Stereotype zu typischen West- und Osteigenschaften vorhanden sind. Bei der Befragung von etwa dreihundert Berliner Studenten zu ihren Partnerwahlpräferenzen wird deutlich, dass Empathie, Erotik und sexuelle Offenheit, ein großer Bekanntenkreis und Geselligkeit sowie ein gutes Verhältnis zur Familie als typisch ostdeutsch wahrgenommen werden. Gutes Benehmen, eine hohe Bildung, beruflicher Erfolg und ökonomische Unabhängigkeit gelten dagegen als typisch westdeutsch. Zudem zeigt sich, dass sich die ost- bzw. westdeutschen Befragten im Hinblick auf politische und kulturelle Merkmale jeweils mehr mit lhresgleichen verbunden fühlen. Dies deutet auf eine Definition der Eigengruppe entlang der Ost-West-Kategorie hin.

Die Journalistin Simone Schmollack (2005) kommt in ihrem Buch zu den „deutsch-deutschen Beziehungen“ allerdings zu einer eher optimistischen Einschätzung, was die "Kompatibilität" von West- und Ostdeutschen angeht, die eine Paarbeziehung miteinander eingehen. Auf der Basis von einigen qualitativen Interviews wird davon berichtet, dass durchaus Konflikte in diesen Partnerschaften auftreten, die sich häufig um das Thema Politik und dabei vor allem um die jüngste deutsche Vergangenheit und Aspekte wie Wende, Arbeitslosigkeit und Treuhand drehen. Unterschiedliche Standpunkte in diesen Fragen seien dabei jedoch weniger auf eine "Ostalgie" bzw. „Westalgie“ zurückzuführen, sondern auf das Bedürfnis, die eigene biografische Identität in der Partnerschaft nicht zu verlieren. Gleichzeitig wird allerdings eine Abschwächung von Ost-West-Divergenzen über die Zeit diagnostiziert: „Ost-West-Paare, die sich bis Mitte der neunziger Jahre getroffen haben, bekamen die kulturellen, emanzipatorischen und ökonomischen Unterschiede stärker zu spüren als Paare, die sich erst vor wenigen Jahren getroffen haben" (Schmollack 2005: 10). Das Fazit auf dem Klappentext des Buches lautet daher: „Nirgendwo ist die deutsch-deutsche Wiedervereinigung offensichtlicher als in der Liebe."

Vor dem Hintergrund dieser widersprüchlichen Befunde besteht das Ziel des vorliegenden Beitrags darin, am Beispiel von Ost-West-Paaren den Widerstreit zwischen überdauernden Sozialisationserfahrungen und Anpassungen an den sozialräumlichen Kontext und neue Interaktionspartner zu untersuchen. Ost-West-Paare stellen insofern eine spezielle Gruppe dar, da das Eingehen einer Partnerschaft mit Personen aus dem "Aufnahmekontext" in der Migrationsforschung als eine fortgeschrittene Assimilationsstufe gilt (Gordon 1964; Alba/Nee 2003). Möglicherweise 
werden Unterschiede zwischen den Partnern folglich im Zuge von Partnerwahl- und Anpassungsprozessen nivelliert. Daneben besteht jedoch die Möglichkeit, dass in der Sozialisation erworbene Prägungen, selbst im Kontext der Binnenmigration und der Aufnahme einer neuen Partnerschaft, stabil bleiben. In diesem Fall könnten Differenzen innerhalb von Ost-West-Partnerschaften zu einer erhöhten Instabilität dieser Partnerschaftsform beitragen. Dies wäre gleichbedeutend mit einer fortwährenden Segmentation sozialer Gruppen entlang der Ost-West-Dimension.

Die Studie ist wie folgt gegliedert: In Abschnitt 2 wird zunächst aus theoretischer Perspektive diskutiert, welche speziellen Charakteristika Ost-West-Paare aufweisen. Dabei wird auf einen theoretischen Rahmen zurückgegriffen, der zwischen drei Einflussgrößen differenziert: der Sozialisation in der ehemaligen DDR bzw. alten BRD, der Adaption der Binnenmigranten an den aktuellen Sozialraum und neue Interaktionspartner sowie der Selektivität von räumlich mobilen Personen (Kulu/ Milewski 2007; Milewski 2009). In Abschnitt 3 werden anschließend die Eigenschaften der Ost-West-Paare in das Rahmenmodell zur Erklärung der Ehestabilität von Lewis und Spanier (1979) integriert. Nach methodischen Anmerkungen im vierten Abschnitt wird im empirischen Teil (Abschnitt 5) zunächst mit Daten des Soziooekonomischen Panels geklärt, wie verbreitet Ost-West-Partnerschaften sind. ${ }^{1} \mathrm{Da}$ bei ist insbesondere auch der Sonderstatus Berlins zu berücksichtigen, da hier die Gelegenheitsstrukturen für die Entstehung von Ost-West-Paaren besonders günstig sind. Die weiteren Analysen befassen sich mit den speziellen Eigenschaften und der Stabilität dieser Partnerschaften. In Abschnitt 6 werden die Ergebnisse schließlich zusammengefasst und diskutiert.

\section{Eigenschaften von Ost-West-Partnerschaften im Kontext von Sozialisations-, Adaptions- und Selektionseffekten}

Die Untersuchung der speziellen Merkmale von Ost-West-Paaren stützt sich auf einen migrationssoziologischen theoretischen Rahmen, der zwischen drei Hypothesen unterscheidet: der Sozialisations-, der Adaptions- und der Selektionshypothese (Kulu/Milewski 2007; Milewski 2009). Im Rahmen der Sozialisationshypothese wird davon ausgegangen, dass individuelle Merkmale wie Erwerbsorientierung oder Religiosität, die durch die Sozialisation in der ehemaligen DDR und der alten BRD bedingt sind, selbst dann unverändert bleiben, wenn der Akteur im Zuge einer Binnenmigration veränderten sozialen Kontextbedingungen ausgesetzt ist. Schneider et al. (1995) gehen in diesem Zusammenhang unter dem Stichwort "divergierende Modernisierung" davon aus, dass sich die unterschiedlichen strukturellen und kulturellen Einflüsse in der alten BRD und der ehemaligen DDR nach der Wiederver-

1 Derzeit fehlen Zahlen zur Verbreitung dieser Partnerschaftsform, da das Statistische Bundesamt im Rahmen der Statistiken zu Eheschließungen nur den aktuellen Wohnort, nicht aber den Ort des Aufwachsens vor 1989 berücksichtigt. (Mitteilung des Statistischen Bundesamtes am 20.11.2012 auf Anfrage) 
einigung nicht rasch auflösen, da ältere Geburtsjahrgänge, vor allem vor dem Jahr 1970, hierdurch in ihrem Denken und Handeln nachhaltig geprägt wurden.

Den Gegenpol zur Sozialisationshypothese stellt die Adaptionshypothese dar. Hiernach wird, im Rahmen von Akkulturations- und Assimilationsprozessen (Gordon 1964; Alba/Nee 2003), eine Anpassung räumlich mobiler Personen an den jeweils aktuellen Lebenskontext postuliert. Hier sind zunächst strukturelle Aspekte des sozial-räumlichen Kontextes zu berücksichtigen, z.B. die Beschäftigungsmöglichkeiten oder die Verfügbarkeit von Einrichtungen zur Kinderbetreuung. Die Entstehung einer Ost-West-Partnerschaft lässt sich darüber hinaus als eine Form von sozialer Assimilation deuten, die in der Forschung zu internationalen Migranten als fortgeschrittene Assimilationsstufe gilt. Beispiele sind das dritte Stadium der Assimilation ("marital assimilation") bei Gordon (1964) oder der Prozess der Grenzverwischung ("boundary blurring") bei Alba und Nee (2003).

Schließlich wird im Rahmen der Selektionshypothese davon ausgegangen, dass die individuellen Merkmale von Binnenmigranten vom Durchschnitt der nicht mobilen Bevölkerung abweichen (Kulu/Milewski 2007; Milewski 2009).

In den folgenden Abschnitten wird vor dem Hintergrund von Sozialisations-, Adaptions- und Selektionseffekten diskutiert, inwieweit sich Ost-West-Paare mit Wohnort in den neuen oder alten Bundesländern von Paaren mit einheitlicher Westoder Ostsozialisation unterscheiden. Bei den untersuchten Merkmalsdimensionen handelt es sich dabei um die ökonomische Lage (Abschnitt 2.1), die Arbeitsteilung des Paares (Abschnitt 2.2), die kirchliche Religiosität (Abschnitt 2.3) und familiale Lebensformen (Abschnitt 2.4). Diese recht heterogene Auswahl orientiert sich vor allem an den spezifischen Langfristfolgen der ehemaligen deutschen Zweistaatlichkeit, die in der Forschung aufgezeigt wurden (Schneider et al. 2012).

\subsection{Zur ökonomischen Lage von Ost-West-Paaren}

Im Hinblick auf die ökonomische Situation des Paares, die sich durch materiellen Wohlstand (Haushaltsäquivalenzeinkommen), die Betroffenheit von Arbeitslosigkeit und subjektive Bewertungen wie die allgemeine Lebenszufriedenheit äußern kann, sollte in erster Linie der aktuelle Wohnort von Bedeutung sein. Für Ost-West-Paare, die in den neuen Bundesländern leben, ist, aufgrund der schlechteren Einkommens- und Beschäftigungschancen, eine stärkere Betroffenheit von ökonomischen Mangelsituationen und Arbeitslosigkeit zu erwarten als für Ost-West-Paare, die in den alten Bundesländern leben. In den Jahrzehnten nach der Wiedervereinigung ist es allerdings zu einer ökonomischen Ost-West-Angleichung gekommen, die sich nach derzeitigem Forschungsstand in verschiedene Phasen einteilen lässt (Göbel et al. 2010): Bezogen auf das Haushaltsäquivalenzeinkommen und die allgemeine Lebenszufriedenheit wurden in der ersten Hälfte der 1990er Jahre deutliche Angleichungsfortschritte erzielt, die sich jedoch zwischen 1995 und 2000 verlangsamt haben. Nach dem Jahrtausendwechsel lassen sich eine erneute Zunahme der Einkommensabstände und eine Stagnation der Zufriedenheitsdifferenzen feststellen. Die ostdeutsche Bevölkerung ist nach wie vor vermehrt im Niedriglohnbereich präsent, stärker von Arbeitslosigkeit betroffen, weist einen höheren Anteil dauerhafter 
Armutsepisoden auf und hat zudem überproportional häufig Einkommenseinbußen im Zuge der Wirtschaftskrisen nach dem Jahr 2000 zu verzeichnen (Göbel et al. 2010: 469). Dementsprechend sollte der sozialräumliche Ost-West-Kontext bis heute mit Unterschieden in der ökonomischen Lage von Paaren verbunden sein.

Unabhängig vom aktuellen Wohnort lässt sich die These aufstellen, dass differierende Wohlfahrtschancen zwischen Partnerschaften auch durch den Ort der Sozialisation bedingt sein können. Ein gutes Beispiel hierfür sind intergenerationale Transfers und Erbschaften. So besteht eine Spätfolge des planwirtschaftlichen Wirtschaftssystems der ehemaligen DDR darin, dass ostdeutsch sozialisierte Personen, aufgrund von geringeren Vermögenswerten und Wohneigentumsquoten in den neuen Bundesländern, nicht nur seltener erben, sondern im Falle einer Erbschaft auch geringere Beträge erhalten (Szydlik/Schupp 2004).

Die selektiven Merkmale der Binnenmigranten sprechen allerdings eher gegen einen generellen ökonomischen Nachteil von Paaren mit ostdeutsch sozialisiertem Partner, da vor allem junge und überdurchschnittlich gut qualifizierte ostdeutsche Personen eine hohe Bereitschaft zur Abwanderung in die alten Bundesländer aufweisen (Windzio 2007; Fuchs-Schündeln/Schündeln 2009). Inwieweit sich OstWest-Paare von Paaren mit übereinstimmender Sozialisation innerhalb des gleichen sozialräumlichen Kontextes unterscheiden, z.B. Ost-West-Paare in Westdeutschland von West-West-Paaren in Westdeutschland, ist somit eine empirische Frage.

\subsection{Zur partnerschaftlichen Arbeitsteilung in Ost-West-Partnerschaften}

Die Sozialisationshypothese lässt erwarten, dass ostdeutsch sozialisierte Frauen eine durchschnittlich höhere Erwerbsorientierung aufweisen als Frauen, die in der alten BRD aufgewachsen sind. Hierfür sind vor allem die divergierenden sozial- und familienpolitischen Arrangements in der DDR und der alten BRD verantwortlich (Trappe 1995). Der westdeutsche Staat hat lange Zeit das Alleinverdienermodell gefördert, wogegen in der DDR frühzeitig die rechtlichen und institutionellen Rahmenbedingungen, z.B. bezogen auf Kinderbetreuungsplätze, geschaffen wurden, um eine schnelle Rückkehr der Frau nach der Familiengründung und eine Vollzeiterwerbstätigkeit beider Geschlechter zu ermöglichen. Sind diese Sozialisationserfahrungen dauerhaft stabil, ist für Ost-West-Paare mit ostdeutsch sozialisierter Frau eine egalitärere Ausrichtung der partnerschaftlichen Arbeitsteilung zu erwarten als für West-West-Paare oder für Ost-West-Paare mit westdeutsch sozialisierter Frau. Im Hinblick auf Selektionseffekte ist zudem bekannt, dass weibliche Ost-West-Migranten überwiegend hoch gebildet und entsprechend erwerbsorientiert sind (FuchsSchündeln/Schündeln 2009).

Darüber hinaus kommt der Adaption an den sozialen Kontext wahrscheinlich eine eigenständige Bedeutung zu. Vor dem Hintergrund der besseren Verfügbarkeit von Kinderbetreuungsplätzen in den neuen Bundesländern (Hank et al. 2004) ist zu erwarten, dass in Ostdeutschland lebende Paare, unabhängig von ihren Sozialisationserfahrungen, im Hinblick auf ihre relative Erwerbsbeteiligung egalitärer ausgerichtet sind als Paare in den alten Bundesländern. Darüber hinaus besteht die Möglichkeit von Akkulturationsprozessen, z.B. im Sinne einer abnehmenden Er- 
werbsorientierung ostdeutsch sozialisierter Frauen im Zuge des Umzugs in die alten Bundesländer und einer Anpassung an einen Partner, der in der alten BRD aufgewachsen ist. Dass sich Geschlechtsrollenorientierungen in Partnerschaften anpassen können, wird durch eine Studie von Kalmijn (2005) mit niederländischen Daten bestätigt. Männer und Frauen werden hiernach über die Zeit egalitärer (traditioneller), je egalitärer (traditioneller) der Partner im Ausgangszustand ist. Das Ausmaß der Anpassung hängt jedoch stark von dem Vorhandensein von Kindern und der Erwerbskonstellation ab. Da bisher keine äquivalenten Studien zu Ost-West-Paaren vorliegen, wird empirisch zu überprüfen sein, ob divergierende Geschlechtsrollenorientierungen in diesen Partnerschaften infolge von Anpassungsprozessen nivelliert werden.

\subsection{Zur Religiosität in Ost-West-Partnerschaften}

Zu den gravierendsten soziokulturellen Ost-West-Differenzen zählt die unterschiedliche Bedeutung von Religion und Kirche. Die alten Bundesländer sind seit jeher durch die süddeutsche katholische Kulturtradition geprägt worden, während in den neuen Bundesländern traditionell die liberalen bis säkularen Strömungen des überwiegend protestantischen Nordostens dominieren (Picke/ 2003). Diese langfristigen Unterschiede in der religiösen Kultur wurden mittelfristig durch die Erfahrungen mit entgegengesetzten politischen Systemen verstärkt (Storch 2003). Als Folge dieser Entwicklungen ist die kirchliche Religiosität in den alten Bundesländern heute noch wesentlich stärker verankert als in den neuen. Bezogen auf die erwachsene Wohnbevölkerung in Privathaushalten liegt der geschätzte Anteil von Kirchenmitgliedern in Westdeutschland im Jahr 2009 bei über $80 \%$ und in Ostdeutschland bereits bei unter 30 \% (Lois 2011). Folglich ist aus Sicht der Sozialisationshypothese zu erwarten, dass sich Ost-West-Paare überdurchschnittlich häufig in ihrer Religiosität unterscheiden. Dies könnte sich dadurch ausdrücken, dass nur der westdeutsch sozialisierte Partner noch konfessionell gebunden und religiös aktiv ist.

Unabhängig von den Sozialisationserfahrungen ist allerdings denkbar, dass nur zwischen religiös ähnlichen Personen Partnerschaften überhaupt zustande kommen. Im Falle der kirchlichen Religiosität haben jedoch Klein und Wunder (1996) gezeigt, dass konfessionelle Homogamie überwiegend nicht Ausdruck von Partnerwahlpräferenzen ist, sondern ein Resultat relativer Gruppengrößen und entsprechender Gelegenheitsstrukturen. Demzufolge hat z.B. eine ostdeutsch sozialisierte, konfessionslose Person, die in die alten Bundesländer zieht, allein aufgrund der Gelegenheitsstrukturen eine größere Chance, Personen mit Konfession zu treffen. Nach der Adaptionshypothese bestünde allerdings die Möglichkeit, dass Unterschiede der Partner im Hinblick auf die Religiosität durch Anpassungsprozesse über die Zeit überwunden werden. Auch diese Annahme wird allerdings durch die bisherige Forschung nur bedingt unterstützt. Lois (2013: 184-209) zeigt am Beispiel der Kirchgangshäufigkeit, dass in Partnerschaften zwar eine Tendenz festzustellen ist, sich über die Zeit an den Partner anzupassen, diese Tendenz aber deutlich schwächer ist als bei Vergleichsmerkmalen wie der Freizeitgestaltung. Den aus der Sozialisation stammenden Prägungen sollte demnach eine relativ große Bedeutung zukommen. 
Schließlich liegen auch bereits erste Ergebnisse dazu vor, inwieweit Ost-WestMigranten in Bezug auf die kirchliche Religiosität selektiv sind. Lois (2013: 173) zeigt, dass Binnenmigranten zwischen Ost- und Westdeutschland, unabhängig von der Mobilitätsrichtung, signifikant häufiger konfessionslos sind und zudem eine signifikant niedrigere Kirchgangshäufigkeit aufweisen als nicht mobile Personen (zu ähnlichen Ergebnissen kommt Vatterrott 2011). Während somit die Sozialisations- und auch die Adaptionshypothese erwarten lassen, dass sich die religiöse Prägung von Partnern in Ost-West-Beziehungen überdurchschnittlich stark unterscheidet, sollte diese Tendenz durch den geschilderten Selektionsmechanismus wieder abgeschwächt werden. Auch die religiöse Ähnlichkeit in Ost-West-Paaren wird damit zu einer empirisch zu klärenden Frage.

\subsection{Zur Wahl familialer Lebensformen in Ost-West-Partnerschaften}

Die bisher diskutierten Ost-West-Unterschiede in den Bereichen ökonomische Lage, Arbeitsteilung und Religiosität sollten auch dazu beitragen, dass die in Ost-West-Beziehungen gewählten familialen Lebensformen vom Durchschnitt der Bevölkerung abweichen. Aus Sicht der Sozialisationsperspektive lassen sich Argumente für eine stärkere Neigung zur Nichtehelichkeit beim ostdeutsch sozialisierten Partner finden. Aus historischer Perspektive ist vor allem die starke Verbreitung von nichtehelichen Geburten im Gebiet der neuen Bundesländer bis in das 19. Jahrhundert zurückzuverfolgen. Diese wurde in der DDR durch die Einführung des Babyjahres, das ledige Mütter privilegierte, verstärkt (Schneider et al. 2012). Trotz der Angleichung sozialrechtlicher Rahmenbedingungen ist die Ost-West-Schere in der Nichtehelichenquote auch nach der Wiedervereinigung größer geworden. Im Jahr 2010 lag der Anteil nichtehelich Geborener an allen Erstgeborenen bei $74 \%$ in Ostdeutschland (ohne Berlin), aber nur bei $37 \%$ in Westdeutschland (Pötzsch 2012).

Zusätzlich ist im Rahmen der Selektionshypothese zu bedenken, dass in der Gruppe der Ost-West-Binnenmigranten Personen mit mindestens einer vorangehenden Scheidung im Lebensverlauf überrepräsentiert sind, vor allem in der ersten Migrationswelle zwischen 1990 und 1995 (Fuchs-Schündeln/Schündeln 2009). Auch dieser Selektionseffekt sollte zu einer geringeren Heiratsneigung in Ost-WestPaaren beitragen, da die Wahrscheinlichkeit des Eingehens einer Zweit- oder Drittehe gegenüber Erstehen geringer ist (Lankuttis/Blossfeld 2003).

Arránz Becker und Lois (2010) finden allerdings insoweit Hinweise auf Adaptionsprozesse, da sich - im Ausgangszustand ledige - Frauen mit Ost-West-Mobilität an das Heiratsmuster westdeutscher Frauen anpassen, obwohl sie soziokulturelle Prägungen ihres ostdeutschen Herkunftskontextes, dazu zählen eine geringe Religiosität und eine relativ ausgeprägte Berufsorientierung, beibehalten. Hierfür könnte eine Anpassung an den westdeutschen Partner verantwortlich gemacht werden, der, aufgrund seiner Sozialisationserfahrungen, eine verstärkte "Eheorientierung" in die Partnerschaft einbringen sollte. Obwohl sich insgesamt mehr Argumente finden lassen, die für eine starke Neigung zur Nichtehelichkeit in Ost-West-Paaren sprechen, handelt es sich hier wiederum um eine empirisch zu klärende Frage. 
Hinsichtlich des Fertilitätsverhaltens sind ebenfalls bleibende Ost-West-Unterschiede nach der Wiedervereinigung zu beobachten. Nach 1990 kam es zwar scheinbar zu einer Anpassung der ostdeutschen Bevölkerung an die neuen strukturellen Rahmenbedingungen. Das Alter bei Familiengründung, das in der DDR deutlich niedriger lag als in der alten BRD, stieg nach 1989 rapide an und führte dadurch kurzfristig zu einem Einbruch der Geburtenziffern (Zapf/Mau 1993). Auch in der Nachwendezeit weisen ostdeutsche Frauen jedoch bei der Geburt des ersten Kindes mit 27.4 Jahren ein etwas geringeres Durchschnittsalter auf als westdeutsche Frauen mit 29.2 Jahren (Pötzsch 2012); zudem ist die lebenslange Kinderlosigkeit in den alten Bundesländern stärker verbreitet als in den neuen (Statistisches Bundesamt 2009).

Im Hinblick auf die Familiengründung ist somit einerseits davon auszugehen, dass in der DDR sozialisierte Personen seltener zu einer dauerhaften Kinderlosigkeit neigen als Personen, die in der alten BRD aufgewachsen sind. Zudem scheinen sich ostdeutsch sozialisierte Personen, die in die alten Bundesländer migrieren, nicht an das westdeutsche Muster einer relativ späten Erstgeburt anzupassen (Vatterrott 2011).

Die speziellen Merkmale der (weiblichen) Ost-West-Migranten, z.B. ihr hohes Bildungsniveau (Fuchs-Schündeln/Schündeln 2009; Windzio 2007), lassen andererseits über einen Selektionseffekt ein häufigeres Auftreten der Kinderlosigkeit in dieser Gruppe erwarten. Die gleiche Schlussfolgerung kann gezogen werden, wenn der Einfluss des jeweiligen westdeutsch sozialisierten Partners berücksichtigt wird, dessen Sozialisationserfahrungen wiederum auf das biografische Modell der späten Familiengründung im Lebenslauf hinauslaufen sollten. Für Ost-West-Paare mit Wohnort in den alten Bundesländern ist ferner der infrastrukturelle Effekt einer geringeren Verfügbarkeit von Kinderbetreuungsangeboten, insbesondere für Kinder unter 3 Jahren (Hank et al. 2004), zu berücksichtigen.

Vor dem Hintergrund dieser komplexen Gemengelage wird auch im Hinblick auf die Familiengründung empirisch zu prüfen sein, inwieweit sich Ost-West-Beziehungen von der Vergleichsgruppe der Paare mit übereinstimmendem Sozialisationshintergrund unterscheiden.

\section{Hypothesen zur Stabilität von Ost-West-Partnerschaften}

Im vorangehenden Abschnitt wurde herausgearbeitet, dass sich Ost-West-Paare wahrscheinlich in Hinblick auf eine Reihe von Merkmalen von Partnerschaften mit einheitlichem Sozialisationskontext unterscheiden. Diese mutmaßlichen Unterschiede müssen jedoch nicht zwangsläufig die Zufriedenheit und die Stabilität einer Paarbeziehung beeinflussen, da sie zum Beispiel in der alltäglichen Kommunikation und Interaktion des Paares nicht relevant sind. Erst wenn sich zeigen lässt, dass OstWest-Paare aufgrund ihrer Charakteristika überdurchschnittlich instabil sind, ist die Schlussfolgerung berechtigt, dass es auch im wiedervereinigten Deutschland noch zu einer Segmentation (Esser 2001: 19-20) sozialer Gruppen entlang der Ost-WestDimension kommt. 
Vor diesem Hintergrund werden nun Hypothesen zum Trennungsrisiko in OstWest-Paaren formuliert. Als theoretische Grundlage dient hier ein mehrdimensionales und integratives Rahmenmodell der Ehestabilität, das von Lewis und Spanier (1979) entwickelt wurde und sowohl gesellschaftliche als auch individuelle und dyadische Merkmale einbezieht. ${ }^{2}$ Die subjektive eheliche Instabilität im Sinne einer Trennungsneigung ist dabei Vorbedingung einer tatsächlich vollzogenen Scheidung und ist selbst wieder von der Ehequalität abhängig. Die Ehequalität wird von sozialen und personalen Ressourcen beeinflusst, die bereits zu Beginn der Ehe festgelegt sind, vom Belohnungswert der partnerschaftlichen Interaktion und von der Zufriedenheit mit dem aktuellen Lebensstil.

Übertragen auf die vorliegende Fragestellung führen die Ost-West-Sozialisationserfahrungen zu spezifischen personalen und sozialen Ressourcen. Der etwas ungenaue Ressourcenbegriff im Modell von Lewis und Spanier (1979) kann unter Rückgriff auf die Familienökonomie (Becker 1981) dahingehend präzisiert werden, dass zwischen komplementären und substituierbaren Ressourcen zu unterscheiden ist. Bei komplementären Merkmalen wie der Religiosität erhöhen sich die Zufriedenheit und Stabilität der Paarbeziehung, wenn die Partner sich möglichst ähnlich sind. Bei substituierbaren Eigenschaften wie der Erwerbsbeteiligung kann dagegen im Rahmen von Spezialisierung und Arbeitsteilung auch eine Unähnlichkeit der Partner effizient sein. Die Einflüsse des aktuellen sozial-räumlichen Kontextes, etwa die unterschiedlichen Einkommens- und Beschäftigungschancen in den alten und neuen Bundesländern, beeinflussen zudem direkt die gegenwärtige Zufriedenheit des Paares mit dem Lebensstil.

$\mathrm{Ob}$ eine Ehe bei geringer Ehequalität und hoher subjektiver Trennungsneigung tatsächlich geschieden wird, hängt im Modell von Lewis und Spanier (1979) zusätzlich davon ab, inwieweit externer Druck zur Aufrechterhaltung der Ehe besteht und wie viele Alternativen zur Ehe zur Verfügung stehen. Auch hier ergeben sich Anknüpfungspunkte zur vorliegenden Fragestellung, da Ost-West-Paare sozialräumlich in großstädtischen Kontexten - vor allem in Berlin - überrepräsentiert sein sollten, weil dort mehr alternative Partner zur aktuellen Partnerschaft zur Verfügung stehen (Schneider et al. 2012). Auf diesen Aspekt wird in Abschnitt 5.1 zurückzukommen sein. Im Folgenden wird zunächst dargestellt, welche Schlussfolgerungen sich für die Stabilität von Ost-West-Paaren ziehen lassen, wenn Unterschiede in der ökonomischen Lage, der partnerschaftlichen Arbeitsteilung, der Religiosität und der familialen Lebensform berücksichtigt werden.

In Abschnitt 2.1 wurde die Hypothese formuliert, dass aktuell in den neuen Bundesländern wohnende Paare, und womöglich auch Paare mit mindestens einem in der ehemaligen DDR aufgewachsenen Partner, in stärkerem Ausmaß von ökonomischen Problemlagen betroffen sind. Hieraus lässt sich schließen, dass die genannten Paare instabiler sind (Deprivations-Hypothese), da sich ökonomische Mangelsituationen sowie Arbeitslosigkeitserfahrungen destabilisierend auf Partnerschaften

2 Eine ausführliche Diskussion sowie Vorschläge zur Weiterentwicklung des Modells finden sich in Arránz Becker (2008: 29-37, 305-320). 
auswirken (im Überblick: Arránz Becker 2008: 32-35). Dies ist vor allem auf negative Veränderungen der Paarinteraktion zurückzuführen. Fühlen sich Paare ökonomisch depriviert, erhöht sich die Wahrscheinlichkeit des Auftretens dysfunktionaler Wahrnehmungen und Verhaltensweisen wie Ängstlichkeit, Depressivität und Demoralisierung. Zudem verringert sich die gegenseitige Unterstützung der Paare und es kommt vermehrt zu Feindseligkeiten und Konflikten (Conger et al. 1990; Conger et al. 1999).

Neben der Ausstattung mit ökonomischen Ressourcen stellt sich die Frage, wie diese in Partnerschaften durch Arbeitsteilung erzeugt werden und welche Konsequenzen dies für die Partnerschaftsstabilität hat. Lewis und Spanier (1979) ordnen diesen Aspekt, wie auch die ökonomische Lage des Paares, der Kategorie "satisfaction with lifestyle" zu. In der Forschung gilt vor allem die Erwerbstätigkeit der Frau als Faktor, der die Trennungswahrscheinlichkeit in Ehen, nicht aber in nichtehelichen Lebensgemeinschaften (Lois 2008), erhöht. Dies wird, wie Böttcher (2006) zusammenfassend dargestellt hat, auf einen verringerten „Ehegewinn“ infolge einer ausbleibenden Effizienzsteigerung durch Arbeitsteilung, eine Doppelbelastung der Frau durch Haus- und Erwerbsarbeit, eine höhere Verfügbarkeit alternativer Partner im Arbeitsumfeld der Frau und den destabilisierenden Selbständigkeitseffekt des Einkommens der Frau zurückgeführt. Der negative Zusammenhang zwischen der Erwerbstätigkeit der Frau und der Ehestabilität wird durch zahlreiche Studien belegt (im Überblick: Wagner/Weiß 2003). Es wird daher die Hypothese formuliert, dass Ost-West-Ehen mit ostdeutsch sozialisierter Frau sowie Ost-Ost-Ehen aufgrund ihrer stärker egalitären Arbeitsteilung instabiler sind als West-West-Ehen oder OstWest-Ehen mit ostdeutsch sozialisiertem Mann (Arbeitsteilungs-Hypothese).

Ein klassisches Beispiel für ein komplementäres Merkmal, das bei Lewis und Spanier (1979) den bereits vorehelich festgelegten Faktoren zugerechnet werden kann, ist die Religiosität. Theoretisch ist hier zu erwarten, dass eine religiöse Ähnlichkeit der Partner positive Paarinteraktionen fördert und das Auftreten von Konflikten unwahrscheinlicher macht (im Überblick: Arránz Becker 2008: 62-71). Bisherige Studien bestätigen diese Annahmen (z.B. Call/Heaton 1997; Wagner/Weiß 2003). Im Ergebnis führt dies zu der Erwartung, dass Ost-West-Paare deshalb instabiler sind als Paare mit übereinstimmendem Sozialisationsort, da sich die religiöse Prägung der Partner überdurchschnittlich oft unterscheidet (Religiöse-Homogamie-Hypothese).

Das theoretische Rahmenmodell von Lewis und Spanier (1979) ist ferner zu einer Zeit entwickelt worden, in der nichteheliche Lebensgemeinschaften (NEL) wenig verbreitet waren. Qian und Lichter (2007) weisen jedoch im Zusammenhang mit interethnischen Partnerschaften darauf hin, dass die Zunahme von NEL die soziale Distanz zwischen sozialen Gruppen verstärken kann, da diese familiale Lebensform, verglichen mit Ehen, allgemein instabiler ist, mit einer geringeren Überlappung der partnerschaftlichen sozialen Netzwerke verbunden ist und zudem weniger häufig mit der Geburt von Kindern einhergeht, die innerhalb der Familienökonomie als beziehungsspezifische Investition gelten. Die stärker ausgeprägte Nichtehelichkeit und Kinderlosigkeit sollte daher zu einer erhöhten Instabilität der Ost-West-Paare beitragen (Nichtehelichkeits-Hypothese, Kinderlosigkeits-Hypothese). 
Ferner ist die in Abschnitt 2.4 diskutierte Selektivität der Ost-West-Binnenmigranten zu berücksichtigen, die überdurchschnittlich häufig bereits einmal geschieden sind. Zweitehen weisen ein erhöhtes Trennungsrisiko auf (Wagner/Weiß 2003). Dies wird vor allem auf die selektiven Merkmale bereits geschiedener Personen, etwa mangelnde Interaktionskompetenzen, zurückgeführt (Arránz Becker 2008: 5960). Daraus folgt die Zweitehen-Hypothese, die ebenfalls eine erhöhte Instabilität der Ost-West-Partnerschaft erwarten lässt.

\section{$4 \quad$ Daten und Methode}

\subsection{Datengrundlage}

Die vorliegende Studie greift auf Daten des Sozio-oekonomischen Panels (SOEP, Wagner et al. 2007) zurück. Für diesen Datensatz spricht, dass dyadische Längsschnittdaten über einen Beobachtungszeitraum von zwei Jahrzehnten (hier: 19902009) zur Verfügung stehen. Die Stichprobe setzt sich aus 16.396 Ehen und nichtehelichen Lebensgemeinschaften (unverheiratete Partner in einem gemeinsamen Haushalt) zusammen, die in den SOEP-Erhebungen A-H seit 1990 beobachtet werden können. 87,2 \% der Beobachtungsepisoden entfallen auf Ehen. Paare mit getrennten Haushalten können nicht berücksichtigt werden, da in diesen Fällen keine Partnerinformationen vorliegen.

In Tabelle 1 ist eine Übersicht über die Fallzahlen, differenziert nach Paartypen, dargestellt. Die Stichprobe umfasst 974 Ost-West-Paare. ${ }^{3}$ Die hier verfügbare Zahl der Ost-West-Paare liegt deutlich höher als in anderen Datensätzen, etwa dem Beziehungs- und Familienpanel, und macht das SOEP derzeit zu der am besten geeigneten Datenquelle für die vorliegende Fragestellung. Allerdings ist der Typ „Ostmann-Westfrau“ in den neuen Bundesländern mit 24 Paaren zu schwach besetzt und wird daher aus den deskriptiven Analysen (Tab. 3 und 4) ausgeschlossen.

In Tabelle 1 ist zusätzlich die prozentuale Verteilung verschiedener Typen von Ost-West-Paaren dargestellt. Zum Beispiel handelt es sich bei 52,7 \% der Ost-WestPaare um den Typ „Ostfrau-Westmann“ mit Wohnort in den alten Bundesländern. Diese Prozentwerte basieren im Gegensatz zu den Absolutzahlen auf gewichteten Berechnungen.

Es wäre ferner möglich, Paare mit übereinstimmendem Sozialisationskontext und Binnenmigration, z.B. Ost-Ost-Paare, die in die alten Bundesländer ziehen, zu analysieren. Dies würde den Rahmen der vorliegenden Arbeit jedoch sprengen. Dasselbe gilt für ethnisch heterogame Paare.

Die Daten werden im Long-Format aufbereitet, sodass auf ein Paar und auf einen Erhebungszeitpunkt jeweils eine Zeile im Datensatz entfällt. Der Datensatz umfasst insgesamt 122.049 Zeilen. Der Beobachtungszeitraum endet im Falle von Panelmor-

3 In 15,2 \% der Fälle wird der Paartyp aufgrund von fehlenden Angaben zum Wohnort vor 1989 imputiert (siehe Tab. A1). 
Tab. 1: Übersicht über die Fallzahlen und die prozentuale Verteilung der OstWest-Paare

\begin{tabular}{lcc}
\hline & \multicolumn{2}{c}{ Aktuelle Region } \\
& $\begin{array}{c}\text { Alte Bundesländer } \\
\text { (inklusive Westberlin) }\end{array}$ & $\begin{array}{c}\text { Neue Bundesländer } \\
\text { (inklusive Ostberlin) }\end{array}$ \\
\hline Gleiche Region vor 1989 & $\begin{array}{c}\text { West-West-Paare } \\
\text { (n }=11.214)\end{array}$ & $\begin{array}{c}\text { Ost-Ost-Paare } \\
\text { (n }=4.208)\end{array}$ \\
Unterschiedliche Regionen vor 1989 & $\begin{array}{c}\text { Ostfrau-Westmann } \\
(\mathrm{n}=563,52,7 \%)\end{array}$ & $\begin{array}{c}\text { Ostfrau-Westmann } \\
\text { (n }=128,17,2 \%)\end{array}$ \\
$\begin{array}{c}\text { Ostmann-Westfrau } \\
(\mathrm{n}=259,27,3 \%)\end{array}$ & $\begin{array}{c}\text { Ostmann-Westfrau } \\
\text { (n }=24,2,8 \%)\end{array}$ \\
\hline
\end{tabular}

Prozentwerte gewichtet mit Querschnitts-Hochrechnungsfaktoren GPHRF-ZPHRF

Quelle: SOEP (1990-2009, eigene Berechnungen)

talität, Rechtszensierung im Jahr 2009 oder einer Trennung der Partner. Dieselbe Person kann über die Zeit in mehreren Partnerschaften vertreten sein.

\subsection{Auswertungsverfahren}

Die empirischen Analysen umfassen zwei Schritte. Zunächst werden verschiedene Merkmale zwischen den Paartypen verglichen. Dabei ist zu berücksichtigen, dass die Ost-West-Paare mit einem mittleren Paaralter von 40,2 Jahren etwa neun Jahre jünger sind als West-West-Paare (49,6 Jahre) bzw. Ost-Ost-Paare (49,2 Jahre). Ein einfacher deskriptiver Vergleich wäre somit wenig informativ, da er, neben den im Mittelpunkt stehenden Sozialisationseffekten, immer auch Altersunterschiede widerspiegeln würde. Aus diesem Grund werden die Eigenschaften der verschiedenen Paare unter Kontrolle von Alters-, Kohorten- und Periodeneffekten verglichen. ${ }^{4}$

Als Analyseverfahren werden zur Analyse der Eigenschaften von verschiedenen Paartypen sog. Random-Effects-(RE)-Modelle (Allison 2009: 21) eingesetzt, die, gewichtet nach dem jeweiligen Varianzanteil, sowohl Unterschiede innerhalb als auch zwischen Paaren berücksichtigen. Da eine der abhängigen Variablen, die Absolutdifferenz der Kirchgangshäufigkeiten der Partner, einen hohen Anteil der Ausprägung 0 aufweist, wird in diesem Fall ein RE-Poisson-Modell berechnet (A/lison 2009: 49-69). Bei dichotomen Variablen kommt ein (binäres oder multinomiales) logistisches Regressionsmodell mit panelrobusten Standardfehlern zum Einsatz.

Der zweite Auswertungsschritt besteht in einer Analyse des relativen Trennungsrisikos in den verschiedenen Paartypen. Hier wird die auf dem Logit-Modell basierende zeitdiskrete Ereignisdatenanalyse herangezogen, die speziell für Paneldaten

4 Die Kontrollvariablen umfassen hier das lineare und quadrierte Alter (Paarmittelwert), DummyVariablen zum jeweiligen Beobachtungszeitraum (1990-1995, 1996-2000, 2001-2005, Referenz: 2006-2009) sowie das als Paarmittelwert operationalisierte Geburtsjahr und das quadrierte Geburtsjahr. 
angemessen ist (Singer/Willett 2003: 357-406). Aus fallzahltechnischen Gründen wird ein gemeinsames Modell für Ehen und NEL geschätzt. Da sich die Logit-Koeffizienten in hierarchischen Regressionsmodellen auch ohne Interkorrelation der unabhängigen Variablen verändern können (Mood 2010), werden hier durchschnittliche Marginaleffekte (average marginal effects, AME) ausgewiesen, auf die dieses Problem nicht zutrifft.

\subsection{Operationalisierungen}

Zu den Operationalisierungen ist vorwegzunehmen, dass alle unabhängigen Variablen um ein Jahr zeitverzögert in die Analysen zur Partnerschaftsstabilität aufgenommen werden. Fehlende Werte werden bei allen unabhängigen Variablen mit Hilfe des Expectation-Maximization-Verfahrens (Allison 2001: 19) imputiert. Im Falle von unregelmäßig gemessenen Variablen erfolgt zuvor eine Fortschreibung bis zur jeweils aktuellen Erhebung. Der Anteil fehlender Werte ist, zusammen mit deskriptiven Statistiken, in Tabelle A1 im Anhang ausgewiesen.

Operationalisierung der Paartypen:

- Die Information, wo die jeweilige Person vor 1989 aufgewachsen ist (alte BRD, DDR) wird der Meta-Datei „PPFAD“ entnommen. Da die „Sozialisationszeit" in der alten BRD bzw. ehemaligen DDR in jüngeren Geburtskohorten kürzer ist, ${ }^{5}$ werden vertiefende Analysen durchgeführt, bei denen die OstWest-Paare in zwei Gruppen (Geburtsjahr der Frau bis 1970 oder nach 1970) eingeteilt werden. Der aktuelle Wohnort (alte und neue Bundesländer) ist zudem in zeitveränderlicher Form für jede Welle verfügbar.

Sozioökonomische Lage und geschlechtsspezifische Arbeitsteilung:

- Der „Index ökonomische Lage“ basiert auf drei Indikatoren, die zunächst zstandardisiert und anschließend durch Mittelwertbildung kombiniert werden (Cronbach's Alpha rangiert zwischen .76 und .82). Zum einen handelt es sich um das bedarfsgewichtete Haushaltsäquivalenzeinkommen (nach neuer OECD-Skala). Da die Forschung zur Partnerschaftsstabilität zeigt, dass weniger eine objektive als subjektiv wahrgenommene ökonomische Deprivation belastend wirkt (Conger et al. 1999), fließen zum anderen Indikatoren zu der Zufriedenheit mit dem Lebensstandard (10-fach abgestuftes Antwortformat) und zu Sorgen um die eigene wirtschaftliche Lage (3-fach abgestuft) ein. Höhere Werte des Index-Wertes entsprechen einer besseren ökonomischen Lage.

- Zusätzlich wird die Arbeitslosigkeit eines Partners oder beider Partner (für mindestens einen Monat pro Jahr) erfasst.

- Zur Abbildung der außerhäuslichen Arbeitsteilung des Paares wird zunächst für jeden Partner ein Erwerbsindex gebildet, der die auf das Jahr hochgerechneten Voll- und Teilzeitmonate wie folgt verrechnet: Vollzeitmonate + (0.5*Teilzeitmonate). Anschließend werden für diesen Index (1.) ein Paarmittelwert sowie (2.) eine gerichtete Partnerdifferenz (Mann-Frau) gebildet (Griffin et al. 1999).

5 Etwa drei Viertel der in Ost-West-Paaren lebenden Personen wurden vor 1974 geboren. Die jüngste Person innerhalb dieses Partnerschaftstyps wurde 1986 geboren. 
- Um eine vom Erwerbsverhalten unabhängige, indirekte Messung der Geschlechtsrollenorientierung zu erhalten, wird auf die Stunden laut Zeitschätzung zurückgegriffen, die der jeweilige Partner am Wochenende mit Hausund Familienarbeit (Waschen, Kochen, Putzen, Kinderbetreuung) verbringt (gemessen in jeder zweiten Welle). Auch hier werden ein Paarmittelwert und ein gerichteter Differenzterm (Mann-Frau) gebildet.

Kirchliche Religiosität:

- Zur Messung der kirchlichen Religiosität wird auf die Indikatoren Kirchgangshäufigkeit (erhoben 1990, 1992, 1994-1999, 2001, 2003, 2005, 2007-2009) und konfessionelle Zugehörigkeit (Messzeitpunkte: 1990, 1997, 2003, 2007) zurückgegriffen. Die Frage zum Kirchgang weist ein vierfach abgestuftes Antwortformat auf ( $4=$ jede Woche, $3=$ jeden Monat, $2=$ seltener, $1=$ nie), das in den durchschnittlichen jährlichen Gottesdienstbesuch (nie $=0$, seltener $=5$, jeden Monat $=12$, jede Woche $=52$ ) umgerechnet wird. Gebildet werden ein Paarmittelwert sowie eine Absolutdifferenz der Partner. Im Falle der Konfessionszugehörigkeit wird - in den Analysen zur Partnerschaftsstabilität - zwischen drei Konstellationen unterschieden: (1.) beide Partner sind konfessionslos, (2.) die Konfessionen der Partner unterscheiden sich bzw. nur ein Partner ist ohne Konfession (Heterogamie) und (3.) die Partner haben die gleiche Konfession (Homogamie).

Familiale Lebensformen:

- Basierend auf den SOEP-Biografiedateien („biobirth“, „biobirthm”, „biomarsy") wird im Bereich der familialen Lebensformen unterschieden, ob es sich um Ehen oder nichteheliche Lebensgemeinschaften handelt (jeweils mit und ohne Scheidungserfahrung, d.h. eine vorangehende Scheidung mindestens eines Partners) und ob beide Partner noch kinderlos sind. In die Analysen zur Partnerschaftsstabilität fließen zudem Zählvariablen zur Anzahl der 0-1-jährigen, 2-4-jährigen und über 4-jährigen Kinder ein, die aktuell, laut der Stellung zum Haushaltsvorstand, im jeweiligen Haushalt leben.

Verweildauern in der zeitdiskreten Ereignisdatenanalyse:

- Im Falle von Ehen wird die Ehedauer als Verweildauerindikator der BiografieDatei "biomarsy" entnommen und, zur Abbildung eines sichelförmig verlaufenden Trennungsrisikos, in der Form eines linearen und logarithmierten Terms operationalisiert.

- $\quad$ Eine Restriktion der SOEP-Daten besteht darin, dass keine Informationen zur Partnerschaftsdauer verfügbar sind, die ein geeigneter Verweildauerindikator für NEL wäre. Als Ersatz wird hier das Lebensalter herangezogen, das typischerweise hoch mit der Partnerschaftsdauer korreliert.

\section{$5 \quad$ Empirische Ergebnisse}

\subsection{Wie hat sich der Anteil von Ost-West-Paaren seit 1990 entwickelt?}

Im ersten Schritt der empirischen Analysen stellt sich die Frage nach der gesellschaftlichen Relevanz des Phänomens "Ost-West-Liebe". In Abbildung 1 ist dargestellt, wie sich die Verbreitung von Ost-West-Paaren seit 1990 entwickelt hat. Die Schätzergebnisse basieren auf gewichteten Daten (SOEP-Querschnittgewichte PHRF), mit denen eine Anpassung an Randverteilungen amtlicher Mikrozensusda- 
Abb. 1: Entwicklung des Anteils von Ost-West-Paaren an allen NEL und Ehen im Zeitraum 1990-2009

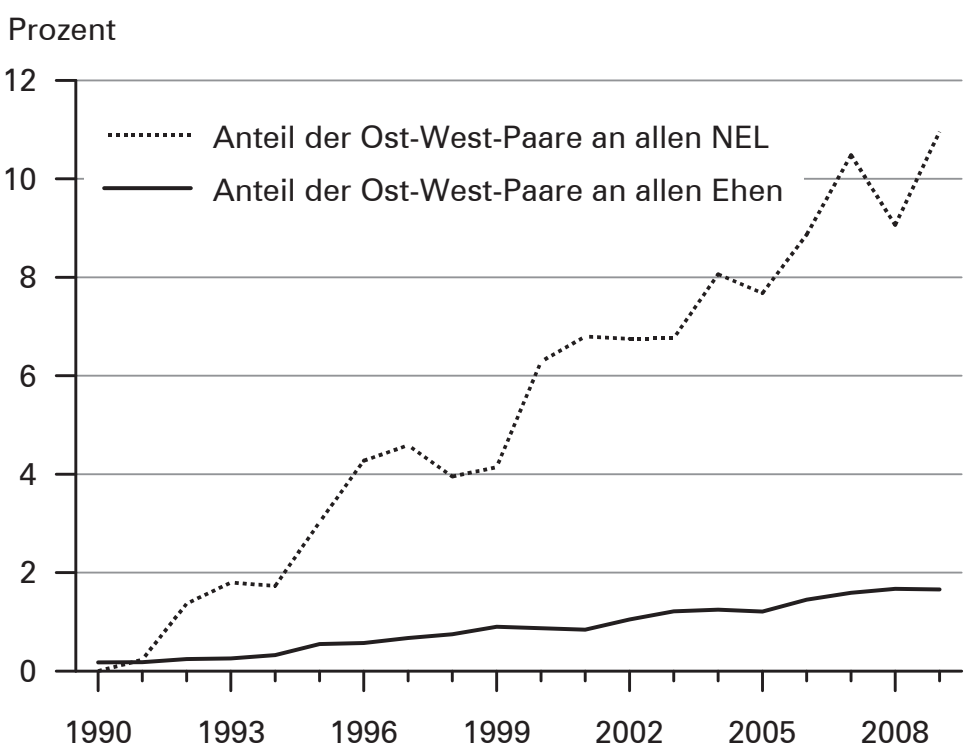

Gewichtung mit Querschnitts-Hochrechnungsfaktoren GPHRF-ZPHRF

Quelle: SOEP (1990-2009, eigene Berechnungen)

ten erfolgt (Göbel et al. 2008). Es zeigt sich ein eindeutiger positiver Trend: Während Partnerschaften zwischen ostdeutsch und westdeutsch sozialisierten Personen im Jahr 1990 praktisch nicht beobachtet werden können, hat sich ihr Anteil an allen Ehen bis zum Jahr 2009 auf 1,7 \% und im Falle der NEL sogar auf 11 \% erhöht.

Die ausgeprägten Unterschiede zwischen Ehen und NEL haben mehrere mögliche Ursachen. Ost-West-Paare hatten überwiegend erst seit 1990 die Gelegenheit, sich zu treffen und zudem sind jüngere Menschen in der Gruppe der Ost-WestBinnenmigranten überrepräsentiert (Fuchs-Schündeln/Schündeln 2009). Der hohe Anteil von NEL ist daher wahrscheinlich zum Großteil einfach auf Alters- und Partnerschaftsdauereffekte zurückzuführen. Darüber hinaus ist in Abschnitt 2.4 bereits die Vermutung formuliert worden, dass Ost-West-Paare, unabhängig vom Alter, gegenüber den West-West-Paaren eine geringere Heiratsneigung aufweisen. Inwiefern dies zutrifft, wird in Abschnitt 5.2 analysiert.

Vertiefende Analysen zeigen zudem, dass es sich bei 3,1\% der Berliner Ehen und bei beträchtlichen 25,3\% der Berliner NEL um Ost-West-Paare handelt, wie durch die günstigen Gelegenheiten des Kennenlernens einfach zu erklären ist.

Zusammenfassend ist festzuhalten, dass die Binnenmigrationsströme zwischen den neuen und alten Bundesländern seit dem Jahr 1990 offensichtlich zu einer zunehmenden Verbreitung von Ost-West-Partnerschaften beigetragen haben. Dieses Ergebnis allein lässt jedoch noch offen, inwieweit eine Einteilung von Partnerschaf- 
ten anhand des Ost-West-Kriteriums sinnvoll ist, weil damit bestimmte soziokulturelle und ökonomische Charakteristika einhergehen. Dieser Frage widmet sich der nun folgende Abschnitt.

\subsection{Die Eigenschaften von Ost-West-Partnerschaften im Vergleich}

In den Tabellen 2 und 3 werden verschiedene Merkmale zwischen den Paartypen verglichen. Um Einflüsse des Sozialisationsortes und des sozial-räumlichen Kontextes soweit wie möglich zu isolieren, werden multivariate Modelle mit einer Reihe von (nicht dargestellten) Kontrollvariablen berechnet.

In Tabelle 2 (Modell 1) wird der Index zur ökonomischen Lage analysiert, der neben dem Haushaltsäquivalenzeinkommen die Zufriedenheit mit dem Lebensstandard und Sorgen über die wirtschaftliche Lage umfasst. Die strukturell besseren Einkommens- und Beschäftigungsmöglichkeiten in Westdeutschland kommen dadurch

Tab. 2: $\quad$ Ökonomische Lage, Arbeitslosigkeit und Arbeitsteilung in nach Sozialisationsregion und aktueller Wohnregion differenzierten Paartypen (b-Koeffizienten mit panelrobusten t- bzw. z-Werten in Klammern)

\begin{tabular}{|c|c|c|c|c|}
\hline & \multicolumn{4}{|c|}{ Modell } \\
\hline & 1 & 2 & 3 & 4 \\
\hline & $\begin{array}{l}\text { Index ökonomische } \\
\text { Lage }\end{array}$ & $\begin{array}{c}\text { Beide AL } \\
\text { (Ref.: keiner) }\end{array}$ & $\begin{array}{c}\text { Einer AL } \\
\text { (Ref.: keiner) }\end{array}$ & $\begin{array}{c}\text { Erwerbsindex } \\
\text { (Mann-Frau) }\end{array}$ \\
\hline & RE linear & multinomiales & Logit-Modell & RE linear \\
\hline \multicolumn{5}{|l|}{ Paartyp } \\
\hline $\begin{array}{l}\text { West-West-Paar, alte BL } \\
\text { (Referenz) }\end{array}$ & - & - & - & - \\
\hline $\begin{array}{l}\text { Ost-Ost-Paar, } \\
\text { neue BL }\end{array}$ & $\begin{array}{l}-.43^{* *} \\
(-40.3)\end{array}$ & $\begin{array}{l}.88^{* *} \\
(17.8)\end{array}$ & $\begin{array}{l}.69^{* *} \\
(21.4)\end{array}$ & $\begin{array}{l}-1.44^{* *} \\
(-19.4)\end{array}$ \\
\hline $\begin{array}{l}\text { Ost(F)-West(M)-Paar, } \\
\text { alte } B L\end{array}$ & $\begin{array}{l}-.07^{* *} \\
(-3.3)\end{array}$ & $\begin{array}{r}.09 \\
(0.7)\end{array}$ & $\begin{array}{l}.14^{+} \\
(1.7)\end{array}$ & $\begin{array}{l}-.32^{*} \\
(-2.0)\end{array}$ \\
\hline $\begin{array}{l}\text { Ost(M)-West(F)-Paar, } \\
\text { alte } B L\end{array}$ & $\begin{array}{l}-.07^{*} \\
(-2.1)\end{array}$ & $\begin{array}{r}-.32 \\
(-1.5)\end{array}$ & $\begin{array}{r}.09 \\
(0.7)\end{array}$ & $\begin{array}{l}-.60^{*} \\
(-2.4)\end{array}$ \\
\hline $\begin{array}{l}\text { Ost }(\mathrm{F}) \text {-West(M)-Paar, } \\
\text { neue BL }\end{array}$ & $\begin{array}{l}-.23^{* *} \\
(-5.4)\end{array}$ & $\begin{array}{l}.68^{*} \\
(2.1)\end{array}$ & $\begin{array}{l}.92^{* *} \\
(5.5)\end{array}$ & $\begin{array}{l}-1.91^{* *} \\
(-5.5)\end{array}$ \\
\hline n (Paare) & 16.372 & & 14.606 & \\
\hline $\mathrm{n}$ (Beobachtungen) & 122.049 & & 101.824 & \\
\hline
\end{tabular}

$+\mathrm{p} \leq .10 ;{ }^{*} \mathrm{p} \leq .05 ;{ }^{*} \mathrm{p} \leq .01$

$\mathrm{RE}=$ Random Effects, $\mathrm{AL}=$ arbeitslos

Modelle 2-4: Beide Partner im erwerbsfähigen Alter

Kontrollvariablen: Lineares und quadriertes Alter (Paarmittelwert); Perioden-Dummys; Geburtsjahr (Paarmittelwert), Geburtsjahr quadriert; familiale Lebensform (Ehe vs. NEL); Anzahl der im Haushalt lebenden Kinder (0-1 Jahre, 2-4 Jahre, über 4 Jahre; nur Modell 4)

Quelle: SOEP (1990-2009, eigene Berechnungen) 
zum Ausdruck, dass die ökonomische Lage von in den neuen Bundesländern lebenden Paaren (Paare der Typen "Ost-Ost" sowie "Ostfrau-Westmann“) insgesamt deutlich schlechter ist als bei Paaren, die in den alten Bundesländern wohnen. Der eigenständige Effekt des sozial-räumlichen Kontextes wird insbesondere dadurch deutlich, dass die Paare des Typs "Ostfrau-Westmann" mit Wohnort in den neuen Bundesländern in einer ökonomisch ungünstigeren Position sind als die entsprechenden Paare, die in den alten Bundesländern leben. Obwohl die Ergebnisse damit eindeutig für einen sozial-räumlichen Adaptionsmechanismus sprechen, sind die Sozialisationserfahrungen nicht ganz zu vernachlässigen. So sind die Ost-West-Paare des Typs "Ostfrau-Westmann" und "Ostmann-Westfrau“ in einer etwas weniger vorteilhaften ökonomischen Situation als die Referenzkategorie der West-West-Paare, mit denen sie den sozial-räumlichen Kontext teilen. Eine Erklärungsmöglichkeit für diesen Befund besteht z.B. darin, dass ostdeutsch sozialisierte Partner Nachteile im Hinblick auf intergenerationale Transfers haben. Darüber hinaus könnten auch von der Binnenmigration selbst kausale Effekte ausgehen, wenn sich z.B. der Erwerbseinstieg und der berufliche Aufstieg eines Partners infolge der Migration verzögern.

Die Ergebnisse zu einer Arbeitslosigkeit eines oder beider Partner (Modelle 2-3 in Tab. 2) fallen ähnlich aus wie in Modell 1, wobei die Bedeutung des sozial-räumlichen Kontextes insgesamt noch etwas deutlicher hervortritt.

Neben der Ausstattung mit materiellen Ressourcen stellt sich die Frage nach der Art und Weise, wie diese im Rahmen der partnerschaftlichen Arbeitsteilung erzeugt werden. Die abhängige Variable in Modell 4 (Tab. 2) ist ein gerichteter Differenzterm, der Auskunft über die außerhäusliche partnerschaftliche Arbeitsteilung gibt. Es handelt sich um die Differenz zwischen den jährlichen Voll- und Teilzeitmonaten des männlichen Partners und denjenigen des weiblichen Partners. Hier deuten hohe Werte auf eine traditionellere Ausrichtung des Paares hin (der Mann arbeitet in höherem Umfang als die Frau). ${ }^{6}$ Für einen fortwährenden Effekt von Sozialisationserfahrungen spricht, dass die in den alten Bundesländern lebenden Ost-West-Paare signifikant egalitärer ausgerichtet sind als die West-West-Paare. Vertiefende Analysen auf Personenebene zeigen, dass hierfür in beiden Fällen die - gegenüber WestWest-Paaren - höhere Erwerbsbeteiligung der in Ost-West-Partnerschaften lebenden Frauen verantwortlich ist. Eine Alternativerklärung zu Sozialisationseinflüssen besteht hier allerdings in einem Selektionseffekt, da weibliche Ost-West-Migranten überwiegend hoch gebildet sind.

Ferner zeigt sich erneut, dass sich Ost-West-Paare des Typs "Ostfrau-Westmann" in Abhängigkeit vom Wohnort deutlich unterscheiden. Die in den neuen Bundesländern lebenden Paare dieser Konstellation praktizieren eine deutlich egalitärere Arbeitsteilung. Hierbei handelt es sich um einen weiteren Hinweis auf einen eigen-

6 Kontrolliert werden hier Boden- und Deckeneffekte durch die Aufnahme der jeweiligen Paarmittelwerte, die in der Form eines linearen und quadrierten Terms einfließen. Wenn z.B. beide Partner nicht erwerbstätig sind, können sie sich, trivialer Weise, nicht im Ausmaß ihrer Erwerbsbeteiligung unterscheiden (Bodeneffekt). 
ständigen Effekt des sozial-räumlichen Kontextes, der in diesem Fall in der besseren Verfügbarkeit von Kinderbetreuungseinrichtungen in den neuen Bundesländern begründet liegen könnte.

Um eigenständige Sozialisationseffekte weiter einzugrenzen, wurden zusätzliche Analysen zur innerhäuslichen Arbeitsteilung am Wochenende durchgeführt, die weniger stark von strukturellen Faktoren, etwa im Hinblick auf verfügbare Kinderbetreuungsplätze und Beschäftigungsangebote, beeinflusst werden sollte. Bei der abhängigen Variablen handelt es sich um einen gerichteten Differenzterm (Mann-Frau) zu den Stunden, die vom jeweiligen Partner für Hausarbeit aufgewendet werden. Die nicht dargestellten Ergebnisse zeigen kaum bedeutsame Unterschiede zwischen den Paartypen. Lediglich Ost-Ost-Paare erweisen sich als signifikant egalitärer als West-West-Paare. Diese Ergebnisse sprechen eher dagegen, dass von den Gelegenheitsstrukturen unabhängige Ost-West-Unterschiede in der Geschlechtsrollenorientierung existieren.

Die in Tabelle 3 dargestellten Analysen widmen sich der Partnerähnlichkeit im Hinblick auf die kirchliche Religiosität sowie die familialen Lebensformen. Die abhängige Variable in Modell 1 nimmt den Wert 1 an, wenn die Partner der gleichen Glaubensgemeinschaft angehören (konfessionelle Homogamie). Unterscheiden sich dagegen die Konfessionen der Partner, oder ist nur ein Partner konfessionslos, wird der Wert 0 vergeben. Die Ergebnisse zeigen, dass sich die konfessionelle Zugehörigkeit der Partner in Ost-West-Beziehungen signifikant häufiger unterscheidet als dies bei West-West-Paaren der Fall ist. Dabei ist unerheblich, ob das Ost-West-Paar aktuell in den neuen oder den alten Bundesländern lebt. Während die Konfessionszugehörigkeit der Partner z.B. bei Ost-West-Paaren mit ostdeutsch sozialisierter Frau und Wohnort in den alten Bundesländern in $50,4 \%$ der Fälle nicht übereinstimmt, trifft dies bei West-West-Paaren nur auf 28,2 \% der Fälle zu.

Darüber hinaus ist in Modell 2 von Interesse, inwieweit sich - bei Kontrolle der konfessionellen Homogamie - Unterschiede in der religiösen Praxis der Partner feststellen lassen. Bei der abhängigen Variablen handelt es sich hier um die ungerichtete Differenz der Kirchgangshäufigkeiten der Partner. Diese Differenz ist innerhalb von Ost-West-Paaren signifikant stärker ausgeprägt als bei West-West-Paaren. Der aktuelle Wohnort spielt dabei nur eine untergeordnete Rolle. Zusammengenommen sprechen die Ergebnisse zur kirchlichen Religiosität damit eindeutig für die fortwährende Bedeutung von divergierenden Sozialisationserfahrungen. Die religiösen Unterschiede innerhalb der Ost-West-Paare können offensichtlich nicht vollständig durch Partnerwahlpräferenzen oder Anpassungsprozesse über die Zeit überwunden bzw. durch Selektionseffekte aufgewogen werden. ${ }^{7}$

7 Zusätzlich sind Unterschiede im Niveau der Religiosität zwischen den verschiedenen Paartypen zu berücksichtigen. So beträgt z.B. die mittlere Häufigkeit von auf das Jahr hochgerechneten Gottesdienstbesuchen bei Ost-West-Paaren mit ostdeutsch sozialisierter Frau und Wohnort in den alten Bundesländern nur 4,0 jährliche Kirchgänge, wogegen sie im Falle von West-WestPaaren bei 10,7 liegt. 
Tab. 3: Kirchliche Religiosität und familiale Lebensformen in nach Sozialisationsregion und aktueller Wohnregion differenzierten Paartypen (b-Koeffizienten mit panelrobusten t- bzw. z-Werten in Klammern)

\begin{tabular}{|c|c|c|c|c|}
\hline & \multicolumn{4}{|c|}{ Modell } \\
\hline & \multirow{3}{*}{$\begin{array}{c}1 \\
\text { Konfessionelle } \\
\text { Homogamie } \\
\text { Logit }\end{array}$} & \multirow{3}{*}{$\begin{array}{c}2 \\
\text { Kirchgang } \\
\text { (|Mann-Frau|) } \\
\text { RE Poisson }\end{array}$} & \multirow{2}{*}{$\begin{array}{l}3 \\
\text { Ehe (1) ver- } \\
\text { sus NEL (0) }\end{array}$} & \multirow{2}{*}{$\begin{array}{c}4 \\
\text { Scheidungs } \\
\text { erfahrung }\end{array}$} \\
\hline & & & & \\
\hline & & & \multicolumn{2}{|c|}{ Logit } \\
\hline \multicolumn{5}{|l|}{ Paartyp } \\
\hline $\begin{array}{l}\text { West-West-Paar, alte BL } \\
\text { (Referenz) }\end{array}$ & - & - & - & - \\
\hline $\begin{array}{l}\text { Ost-Ost-Paar, } \\
\text { neue BL }\end{array}$ & $\begin{array}{l}-.75^{* *} \\
(-13.6)\end{array}$ & $\begin{array}{l}-.06^{* *} \\
(-3.6)\end{array}$ & $\begin{array}{l}-.33^{* *} \\
(-6.1)\end{array}$ & $\begin{array}{r}.04 \\
(0.8)\end{array}$ \\
\hline $\begin{array}{l}\text { Ost(F)-West(M)-Paar, } \\
\text { alte } B L\end{array}$ & $\begin{array}{l}-.88^{* *} \\
(-7.6)\end{array}$ & $\begin{array}{l}.07^{* *} \\
(3.0)\end{array}$ & $\begin{array}{l}-1.39^{* *} \\
(-10.6)\end{array}$ & $\begin{array}{l}1.45^{* *} \\
(11.5)\end{array}$ \\
\hline $\begin{array}{l}\text { Ost(M)-West(F)-Paar, } \\
\text { alte } B L\end{array}$ & $\begin{array}{l}-.46^{* *} \\
(-3.1)\end{array}$ & $\begin{array}{l}.09^{*} \\
(2.4)\end{array}$ & $\begin{array}{l}-1.30^{* *} \\
(-7.0)\end{array}$ & $\begin{array}{l}1.74 * * \\
(10.0)\end{array}$ \\
\hline $\begin{array}{l}\text { Ost }(F) \text {-West(M)-Paar, } \\
\text { neue } B L\end{array}$ & $\begin{array}{l}-.78^{* *} \\
(-3.9)\end{array}$ & $\begin{array}{l}.21^{* *} \\
(4.3)\end{array}$ & $\begin{array}{l}-2.38^{* *} \\
(-8.1)\end{array}$ & $\begin{array}{l}1.60^{* *} \\
(6.1)\end{array}$ \\
\hline n (Paare) & 14.970 & & 16.372 & \\
\hline n (Beobachtungen) & 97.121 & & 122.049 & \\
\hline
\end{tabular}

$+\mathrm{p} \leq .10 ;{ }^{*} \mathrm{p} \leq .05 ;{ }^{* *} \mathrm{p} \leq .01$

$\mathrm{RE}=$ Random Effects, NEL $=$ Nichteheliche Lebensgemeinschaft

Modell 1 ohne homogam konfessionslose Paare

Kontrollvariablen: Lineares und quadriertes Alter (Paarmittelwert); Perioden-Dummys; Geburtsjahr (Paarmittelwert), Geburtsjahr quadriert; familiale Lebensform (Ehe vs. NEL; nur Modelle 1 und 2), Paarmittelwert Religiosität linear und quadriert und Dummy zu konfessioneller Homogamie (nur Modell 2); Anzahl der im Haushalt lebenden Kinder (0-1 Jahre, 2-4 Jahre, über 4 Jahre; nur Modell 3)

Quelle: SOEP (1990-2009, eigene Berechnungen)

In Modell 3 wird weiterhin für die verschiedenen Paartypen untersucht, ob es sich um eine nichteheliche Lebensgemeinschaft (codiert mit 0) oder Ehe (codiert mit 1) handelt. In Modell 4 nimmt die abhängige Variable den Wert 1 an, wenn mindestens ein Partner bereits eine Scheidung erlebt hat.

Die Ergebnisse in Modell 3 verdeutlichen, dass Ost-West-Paare, auch bei Kontrolle von Alterseffekten und dem Vorhandensein von Kindern, deutlich stärker zu nichtehelichen Lebensgemeinschaften neigen als West-West-Paare mit der insgesamt stärksten Ehe-Orientierung. Die Heiratsneigung in den Ost-West-Beziehungen ist dabei, unabhängig vom aktuellen Wohnort, noch einmal deutlich niedriger als bei Ost-Ost-Partnerschaften. Da Alterseffekte kontrolliert sind, deutet dieser Befund zumindest auf Verzögerungen im Institutionalisierungsprozess bei Ost-West-Beziehungen hin (Kopp et al. 2010). Eine einfache Erklärung, die aufgrund von Daten- 
restriktionen nicht getestet werden kann, besteht darin, dass Ost-West-Paare erst ab 1990 Gelegenheit hatten, sich zu treffen, und daher wahrscheinlich eine durchschnittlich kürzere Partnerschaftsdauer aufweisen. Die eigenständige Bedeutung des sozial-räumlichen Kontextes kommt darüber hinaus wiederum dadurch zum Ausdruck, dass sich in den neuen und alten Bundesländern lebende Paare des Typs "Ostfrau-Westmann" unterscheiden, da die Heiratsneigung der in Ostdeutschland lebenden Paare vergleichsweise geringer ist.

In Modell 4 wird zudem deutlich, dass in Ost-West-Paaren überproportional häufig bereits mindestens ein Partner eine Scheidung erlebt hat. Zusatzanalysen zeigen, in Übereinstimmung mit der Forschung (Fuchs-Schündeln/Schündeln 2009), dass dies vor allem auf den migrierten Partner zutrifft. Nach vertiefenden Analysen ist die Selektivität der Ost-West-Paare im Hinblick auf Scheidungserfahrungen auch zum Teil für ihre geringe Heiratsneigung verantwortlich, die Modell 3 dokumentiert.

In nicht dargestellten Analysen wurde zudem der Frage nachgegangen, ob sich das Niveau der Kinderlosigkeit bei Ost-West-Paaren, unter Kontrolle der Lebensformen NEL und Ehe, signifikant von West-West-Paaren unterscheidet. Die Ergebnisse zeigen, dass hier, im Widerspruch zur Kinderlosigkeits-Hypothese, keine statistisch bedeutsamen Unterschiede festzustellen sind. Lediglich Ost-Ost-Paare sind erwartungsgemäß signifikant seltener kinderlos als die Referenzgruppe der West-WestPaare.

Um kohortenspezifische Unterschiede zu testen, wurden ferner alle in den Tabellen 2 und 3 dargestellten Ergebnisse zwischen zwei Gruppen verglichen: für Paare, in denen die Frau bis 1970 oder nach 1970 geboren wurde. Für die ökonomische Lage, die innerhäusliche und außerhäusliche Arbeitsteilung sowie die kirchliche Religiosität zeigen sich keine signifikanten Differenzen. Hinsichtlich der Heiratsneigung und dem Vorhandensein von Scheidungserfahrungen sind allerdings Kohorteneffekte nachweisbar, da die Heiratsneigung bei Ost-West-Paaren jüngerer Geburtskohorten zunimmt und der Anteil von Personen, die bereits eine Scheidung erlebt haben, abnimmt. Eine Erklärung für diese Befunde besteht darin, dass sich die selektiven Merkmale der Ost-West-Binnenmigranten im Laufe der Zeit nachweislich verändert haben. Ostdeutsch sozialisierte Personen, die in der zweiten großen Migrationswelle zwischen 1997/1998 und 2004/2005 ihren Wohnort in die alten Bundesländer verlegt haben, sind, verglichen mit der ersten Migrationswelle zu Beginn der 1990 er Jahre, jünger und weisen seltener bereits Scheidungserfahrungen auf, die sich hemmend auf eine Wiederverheiratung auswirken könnten (Fuchs-Schündeln/ Schündeln 2009).

\subsection{Wie stabil sind Ost-West-Paare?}

Als Zwischenfazit der Analysen im vorangehenden Abschnitt lässt sich festhalten, dass Ost-West-Paare wie erwartet einige „Risikofaktoren“ aufweisen, die potentiell zu einer erhöhten Instabilität beitragen könnten. Dabei handelt es sich z.B. um die Differenzen in der kirchlichen Religiosität oder die ausgeprägte Neigung zur Nichtehelichkeit. Mit den in Tabelle 4 dargestellten Ereignisdatenanalysen wird nun ab- 
schließend getestet, inwieweit sich das Trennungsrisiko zwischen den verschiedenen Paartypen unterscheidet und wodurch diese Differenzen erklärbar sind.

Als Ausgangspunkt ist auf Basis von Modell 1 festzustellen, dass die bedingte Wahrscheinlichkeit einer Trennung bei Ost-West-Paaren mit Wohnort in den alten Bundesländern um 1,2 Prozentpunkte und bei Ost-West-Paaren, die in den neuen Bundesländern leben, um 1,5 Prozentpunkte höher liegt als bei der Referenzkategorie der West-West-Paare. ${ }^{8}$ Beide Unterschiede sind hochsignifikant und, da es sich um Prozentpunkte handelt, auch von nicht unerheblicher Größenordnung. Ein weiteres Beispiel kann dies verdeutlichen: Die Wahrscheinlichkeit einer Trennung liegt bei einem durchschnittlichen Paaralter von 30 Jahren bei allen Ost-West-Paaren zusammengenommen mit etwa $5 \%$ doppelt so hoch wie bei der stabilsten Gruppe, den West-West-Paaren (2,5\% Trennungswahrscheinlichkeit in diesem Alter).

Zur Erklärung dieser Differenzen werden nun schrittweise Variablen in das Regressionsmodell eingeführt, die sich verschiedenen Merkmalsdimensionen zuordnen lassen. In Modell 2 wird zunächst ein sozialräumlicher Selektionsmechanismus berücksichtigt. In Abschnitt 4.1 wurde bereits festgestellt, dass Ost-West-Paare in Berlin überproportional häufig vertreten sind. Zudem zeigt sich in Modell 2, dass das Trennungsrisiko in großstädtischen Kontexten wie Berlin, wahrscheinlich auch infolge einer stärkeren Verfügbarkeit alternativer Partner, erhöht ist (Arránz Becker 2008: 41-44). Die Koeffizienten für Ost-West-Paare gehen jedoch bei Kontrolle des Berlin-Dummys in Modell 2 nur sehr geringfügig zurück.

In Modell 3 werden familiale Lebensformen durch die Berücksichtigung der im Haushalt lebenden Kinder sowie durch die Unterscheidung zwischen NEL und Ehen, jeweils mit und ohne Scheidungserfahrung eines Partners, kontrolliert. In Übereinstimmung mit der Forschung zeigen sich stabilisierende Effekte der ehelichen gegenüber der nichtehelichen Lebensform (Kopp et al. 2010); gleichzeitig sind jeweils Paare, bei denen mindestens ein Partner bereits eine Scheidung erlebt hat, instabiler (Wagner/Weiß 2003). Darüber hinaus bestätigt sich auch der stabilisierende Effekt kleiner Kinder (im Altersbereich 0-1 Jahre). Die Nettounterschiede zwischen Ost-West-Paaren und West-West-Paaren gehen bei Kontrolle der familialen Lebensformen deutlich zurück und sind, im Falle von Ost-West-Paaren mit Wohnort in den neuen Bundesländern, nicht mehr signifikant. Nach vertiefenden Analysen ist diese starke Effektreduzierung vor allem auf die stärkere Prävalenz von NEL und Ehen höherer Ordnung bei Ost-West-Paaren zurückzuführen. Hierin kann ein starker Beleg für die Zweitehen-Hypothese und die Nichtehelichkeits-Hypothese gesehen werden.

Im vierten Modell fließen Indikatoren ein, die neben der ökonomischen Lage des Paares die geschlechtsspezifische Arbeitsteilung abbilden. Es kann bestätigt werden, dass Paare mit günstiger ökonomischer Lage stabiler sind $(b=-.003)$ und sich zudem die Arbeitslosigkeit eines Partners destabilisierend auswirkt. Im Hinblick auf die außerhäusliche Arbeitsteilung zeigt der konditionale Haupteffekt des Differenzterms "Erwerbsindex Mann - Erwerbsindex Frau“ (AME = -.0004), dass Ehen ohne

8 Ost-West-Paare in den alten und neuen Bundesländern werden aus Fallzahlgründen jeweils zu einer Gruppe zusammengefasst. 
Tab. 4: Zeitdiskrete Ereignisdatenanalyse zum Trennungsrisiko in nach aktueller Wohnregion und Sozialisationsregion differenzierten Paartypen (durchschnittliche Marginaleffekte mit z-Werten in Klammern)

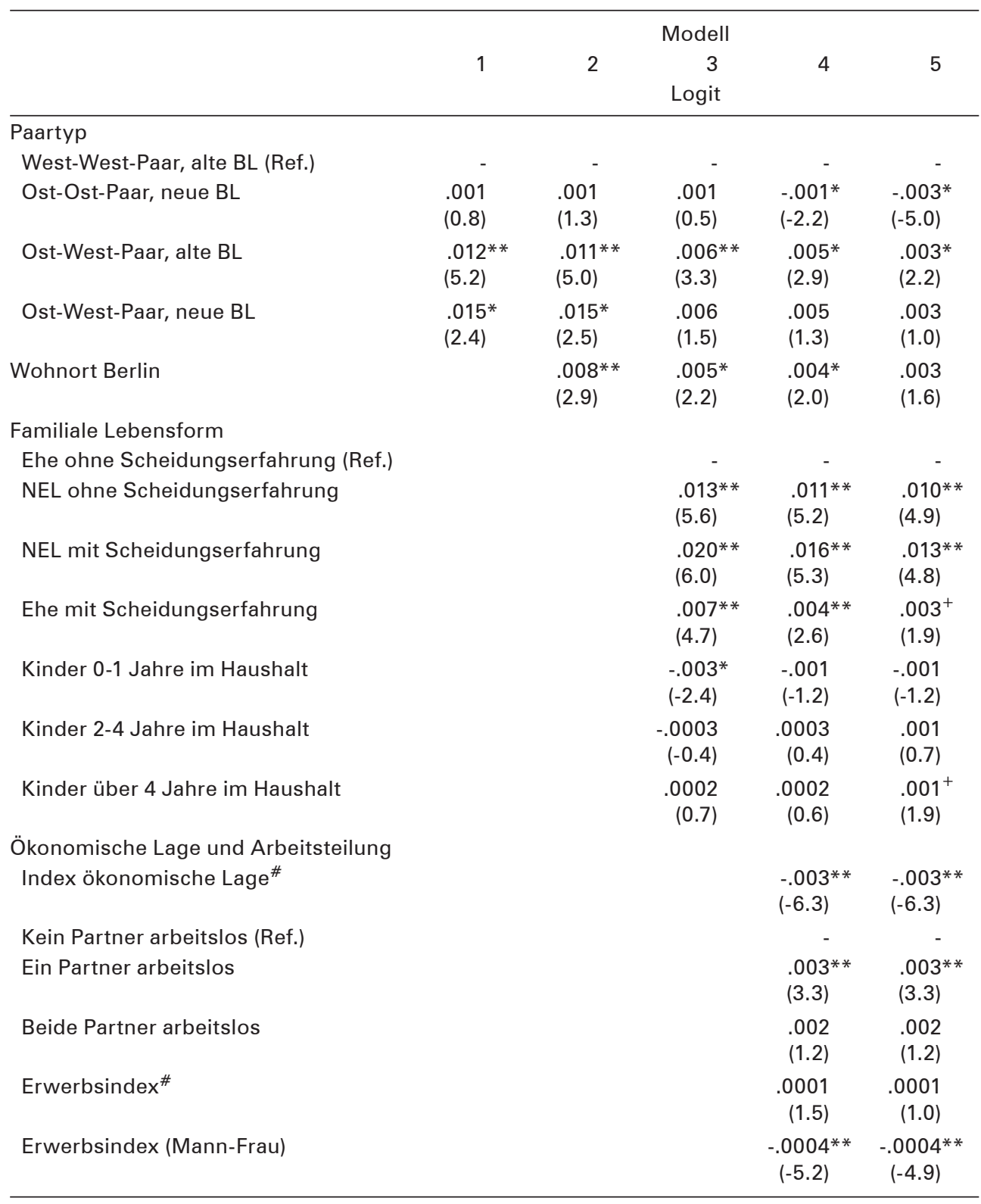


Tab. 4: $\quad$ Fortsetzung

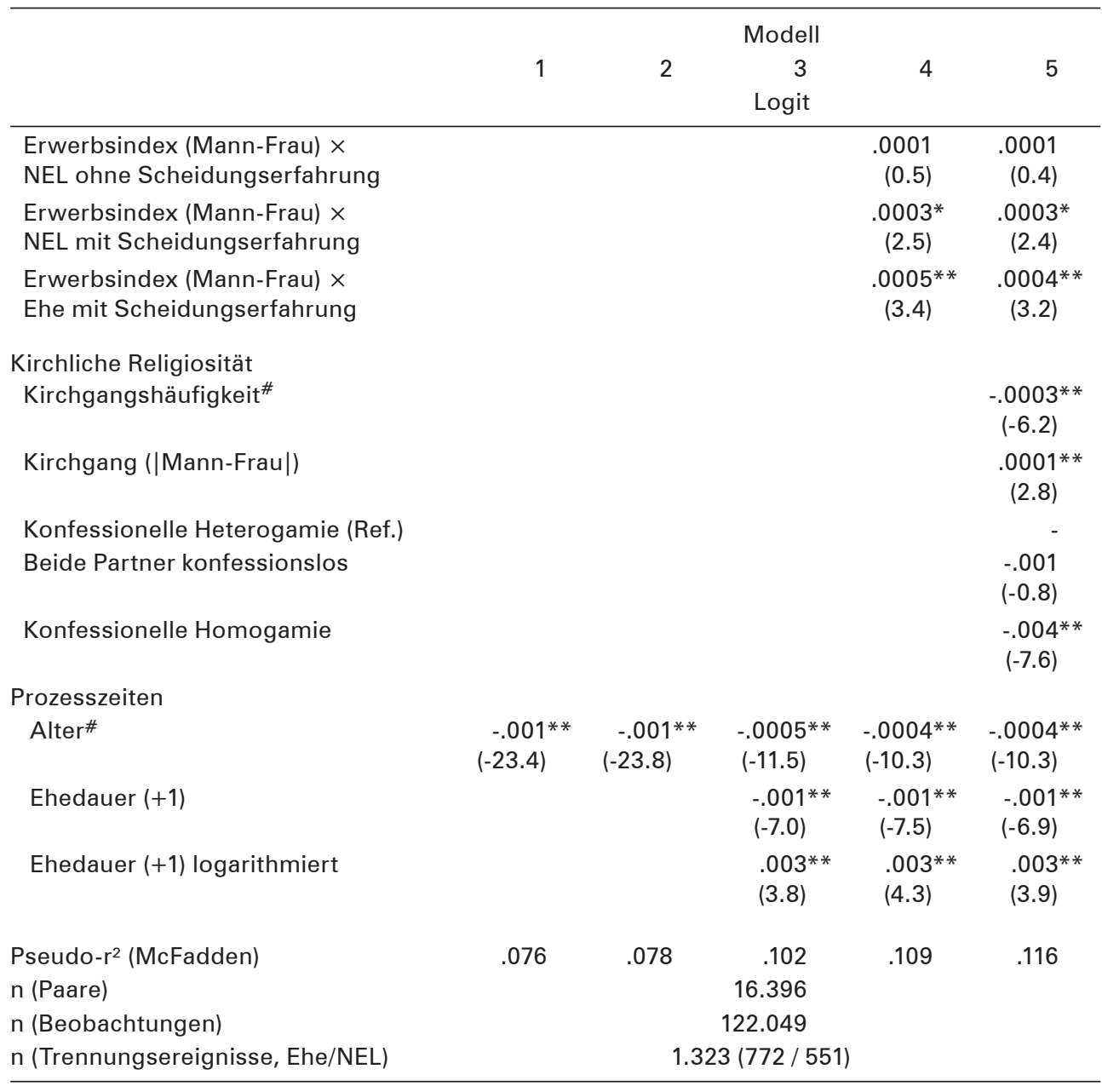

** $\mathrm{p} \leq .01 ; * \mathrm{p} \leq .05 ;+\mathrm{p} \leq .10$

$\mathrm{BL}=$ Bundesländer, NEL $=$ Nichteheliche Lebensgemeinschaft

\# = operationalisiert als Paarmittelwert

Quelle: SOEP (1990-2009, eigene Berechnungen)

Scheidungserfahrung durch eine traditionelle Arbeitsteilung stabilisiert werden. Wie die signifikanten Interaktionseffekte dokumentieren, ist dieser Zusammenhang jedoch weder auf Ehen noch auf NEL, in denen mindestens ein Partner bereits geschieden war, übertragbar. Der multivariat verbleibende Unterschied zwischen OstWest-Paaren in den alten Bundesländern und West-West-Paaren geht insgesamt nur geringfügig im Vergleich der Modelle 3 und 4 zurück. Über die ökonomische 
Lage und die Arbeitsteilung der Partner ist die erhöhte Instabilität der Ost-WestPartnerschaften, im Widerspruch zur Deprivations- und Arbeitsteilungs-Hypothese, somit kaum erklärbar.

Abschließend wird in Modell 5 die kirchliche Religiosität berücksichtigt. Erwartungsgemäß sind Paare, die im Mittel relativ häufig einen Gottesdienst besuchen, stabiler (AME = -.0003; Niveaueffekt). Zudem erhöht sich das Risiko einer Trennung, wenn die Konfessionszugehörigkeit der Partner nicht übereinstimmt bzw. wenn Unterschiede in der religiösen Praxis vorliegen (AME $=-.004$ für konfessionelle Homogamie und $\mathrm{AME}=.0001$ für die ungerichtete Differenz der Kirchgangshäufigkeiten). Unterschiede im Bereich der Religiosität sind für die Stabilität der Ost-West-Paare nicht ganz unerheblich: Im Vergleich der Modelle 4 und 5 ist ein Rückgang des Effekts für Ost-West-Paare in den alten Bundesländern zu beobachten (von .005 auf .003). Die Religiöse-Homogamie-Hypothese wird durch diesen Befund zumindest tendenziell unterstützt.

\section{$6 \quad$ Zusammenfassung und Diskussion}

Als ein erstes Hauptergebnis der vorliegenden Untersuchung ist festzuhalten, dass Partnerschaften zwischen einer westdeutsch und einer ostdeutsch sozialisierten Person quantitativ immer stärker verbreitet sind. Infolge der Binnenmigrationsströme seit der Wiedervereinigung hat sich der geschätzte Anteil der Ost-West-Paare an allen Ehen bzw. nichtehelichen Lebensgemeinschaften im Jahr 2009 auf 1,7 \% bzw. $11 \%$ erhöht. Diese Partnerschaftsform ist zudem in den alten Bundesländern und insbesondere in Berlin überproportional häufig anzutreffen.

Zahlen zur Inzidenz der Ost-West-Paare beantworten jedoch noch nicht die Frage, inwieweit es sinnvoll ist, Paarbeziehungen nach dem Kriterium des aktuellen Wohnortes in den alten und neuen Bundesländern und nach ihrem Sozialisationsort zu differenzieren. Die weiteren Analysen bestätigen jedoch, dass mit dem derzeitigen sozial-räumlichen Kontext und dem Ort des Aufwachsens systematische Unterschiede im Hinblick auf die ökonomische Wohlfahrt des Paares sowie die soziokulturellen Prägungen der Partner einhergehen. Eigenständige Kontexteinflüsse kommen vor allem dadurch zum Ausdruck, dass sich bei Konstanthaltung der Sozialisationserfahrungen des Paares deutliche sozialräumliche Unterschiede zeigen. So weisen Ost-West-Paare mit ostdeutsch sozialisierter Frau und westdeutsch sozialisiertem Mann, die in den neuen Bundesländern leben, eine schlechtere ökonomische Lage, eine egalitärere Arbeitsteilung und eine geringere Heiratsneigung auf als die in den alten Bundesländern wohnenden Paare dieses Typs.

Gleichzeitig finden sich Hinweise darauf, dass sozialisierte Prägungen auch im Zuge von Binnenmigration und sozialer Assimilation nicht vollständig nivelliert werden. Dies kommt z.B. dadurch zum Ausdruck, dass in den alten Bundesländern lebende Ost-West-Paare durch eine ungünstigere ökonomische Lage und eine egalitärere außerhäusliche Arbeitsteilung gekennzeichnet sind als die innerhalb des gleichen sozialräumlichen Kontextes lebenden West-West-Paare. Das deutlichste Beispiel für die Bedeutung der Sozialisation sind darüber hinaus die Unterschie- 
de in der kirchlichen Religiosität, die innerhalb von Ost-West-Paaren bestehen. Die Ergebnisse zeigen hier, dass sowohl die Konfessionszugehörigkeit als auch die Gewohnheiten in der Häufigkeit von Gottesdienstbesuchen zwischen den Partnern in Ost-West-Beziehungen überproportional häufig nicht übereinstimmen.

Einschränkend ist darauf hinzuweisen, dass Alternativerklärungen für die genannten Befunde, die sich auf die Selektivität der Binnenmigranten beziehen, nicht ausgeschlossen werden können. Zum Beispiel ist die relativ egalitäre Arbeitsteilung der Ost-West-Paare potentiell auch darauf zurückzuführen, dass weibliche Binnenmigranten hoch gebildet sind und eine entsprechend hohe Berufsorientierung aufweisen. Um Selektions- und Adaptionseffekte vollständig zu differenzieren, wäre es in einem erweiterten Analysedesign notwendig, Binnenmigranten vor und nach der Migration zu beobachten.

Ein weiteres wichtiges Ergebnis des vorliegenden Beitrags besteht darin, dass die bisher diskutierten Eigenschaften der Ost-West-Paare Konsequenzen für die Stabilität dieser Partnerschaftsform haben. Ost-West-Paare sind signifikant instabiler als west- und ostdeutsche Paare mit übereinstimmendem Sozialisationsort. Die Ereignisdatenanalysen haben verdeutlicht, dass diese erhöhte Instabilität teilweise auf Divergenzen in der konfessionelle Zugehörigkeit und der religiösen Praxis zurückzuführen ist. Im Hinblick auf die kirchliche Religiosität ist folglich eine kulturelle Distanz zwischen westdeutsch und ostdeutsch sozialisierten Personen festzustellen, die über eine erhöhte Instabilität von Paarbeziehungen tendenziell auch zu einer Verfestigung sozialer Gruppengrenzen entlang der Ost-West-Dimension beiträgt. Konfession und Kirchgangshäufigkeit sind allerdings lediglich indirekte Indikatoren für die zugrundeliegenden Werte und Lebensstilelemente. Daher müsste in vertiefenden Analysen gezeigt werden, wie sich religiöse Differenzen konkret auf die Paarinteraktion auswirken.

In überraschender Deutlichkeit ist die Hauptursache für das erhöhte Trennungsrisiko der Ost-West-Beziehungen jedoch im Bereich der familialen Lebensformen und der Beziehungsbiografie zu suchen: Ost-West-Paare trennen sich vor allem deshalb relativ häufig, da sie oft unverheiratet sind und zudem mindestens ein Partner häufig bereits einmal geschieden war. Die geringe Heiratsneigung ist potentiell auf die durchschnittlich kürzere Partnerschaftsdauer der Ost-West-Paare zurückzuführen, die in der Regel erst seit der Wiedervereinigung die Gelegenheit hatten, sich zu treffen. Der auffällig hohe Anteil von Personen mit Scheidungserfahrung in dieser Bevölkerungsgruppe erklärt sich dagegen höchstwahrscheinlich über einen Selektionseffekt, da bereits geschiedene Personen nach vorliegenden Forschungsergebnissen eine höhere Wahrscheinlichkeit aufweisen, innerhalb Deutschlands zu migrieren.

Für die Zukunft stellt sich die Frage, ob die aufgezeigten Besonderheiten der OstWest-Paare in jüngeren Kohorten verschwinden. Dies wäre der Fall, wenn sich diese Gruppe an das „Durchschnittspaar" der Allgemeinbevölkerung angleicht. Auch der umgekehrte Fall ist nicht ganz unplausibel. In gewisser Hinsicht sind die Ost-WestBeziehungen „ihrer Zeit voraus", da einige auffällige Parallelen zwischen ihren speziellen Eigenschaften - egalitäre Arbeitsteilung, Nichtehelichkeit, konfessionelle Heterogamie - und verschiedenen allgemeinen Trends festzustellen sind. Dazu zählen 
der Anstieg des Heiratsalters und die Zunahme nichtehelicher Lebensgemeinschaften (Peuckert 2008: 32-47), die sich erhöhende Erwerbsbeteiligung der Frau (Peuckert 2008: 229-283) und der Anstieg des Anteils von konfessionellen Mischehen (Klein/Wunder 1996). Aus diesem Grund bleibt auch im Zusammenhang mit Paarbeziehungen die Frage spannend, inwieweit der innerdeutsche Ost-West-Vergleich infolge des allgemeinen Modernisierungstrends zunehmend obsolet wird.

\section{Literatur}

Alba, Richard D.; Nee, Victor 2003: Remaking the American mainstream: Assimilation and contemporary immigration. Cambridge, MA: Havard University Press.

Allison, Paul D. 2001: Missing data. Quantitative applications in the social sciences 136. Thousand Oaks: Sage.

Allison, Paul D. 2009: Fixed effects regression models. Quantitative applications in the social sciences 160 . Thousand Oaks: Sage.

Arránz Becker, Oliver 2008: Was hält Partnerschaften zusammen? Psychologische und soziologische Erklärungsansätze zum Erfolg von Paarbeziehungen. Wiesbaden: VS Verlag.

Arránz Becker, Oliver; Lois, Daniel 2010: Unterschiede im Heiratsverhalten westdeutscher, ostdeutscher und mobiler Frauen. Zur Bedeutung von Transformationsfolgen und soziokulturellen Orientierungen. In: Soziale Welt 61: 5-27 [doi: 10.5771/00386073-2010-1-5].

Becker, Gary S. 1981: A treatise on the family. Cambridge/London: Harvard University Press.

Böttcher, Karin 2006: Scheidung in Ost- und Westdeutschland. Der Einfluss der Frauenerwerbstätigkeit auf die Ehestabilität. In: KöIner Zeitschrift für Soziologie und Sozialpsychologie 58,4: 592-616 [doi: 10.1007/s11577-006-0258-1].

Call, Vaughn R. A.; Heaton, Tim B. 1997: Religious influence on marital stability. In: Journal for the Scientific Study of Religion 36,3: 382-392 [doi: 10.2307/1387856].

Conger, Rand D. et al. 1990: Linking economic hardship to marital quality and instability. In: Journal of Marriage and the Family 52: 643-656.

Conger, Rand D.; Rueter, M. A.; Elder Jr., Glen H. 1999: Couple resilience to economic pressure. In: Journal of Personality and Social Psychology 76,1: 54-71 [doi: 10.1037//0022-3514.76.1.54].

Esser, Hartmut 2001: Integration und ethnische Schichtung. Arbeitspapiere des Mannheimer Zentrums für Europäische Sozialforschung 40. Mannheim.

Fuchs-Schündeln, Nicola; Schündeln, Matthias 2009: Who stays, who goes, who returns? East-West migration within Germany since reunification. In: Economics of Transition 17: 703-738 [doi: 10.1111/j.1468-0351.2009.00373.x].

Göbel, Jan; Habich, Roland; Krause, Peter 2010: Ost-West-Angleichung von Einkommen und Zufriedenheit im Lebenszyklus. In: Krause, Peter; Ostner, Ilona (Hrsg.): Leben in Ost- und Westdeutschland. Eine sozialwissenschaftliche Bilanz der deutschen Einheit 1990-2010. Frankfurt/M./New York: Campus.

Göbel, Jan et al. 2008: Daten- und Datenbankstruktur der Längsschnittstudie Soziooekonomisches Panel (SOEP). In: SOEP Papers 89: 1-42. 
Gordon, Milton M. 1964: Assimilation in American life: The role of race, religion, and national origins. Oxford: Oxford University Press.

Griffin, Dale; Murray, Sandra; Gonzalez, Richard 1999: Difference score correlations in relationship research: A conceptual primer. In: Personal Relationships 6: 505-518 [doi: 10.1111/j.1475-6811.1999.tb00206.x].

Hank, Karsten; Kreyenfeld, Michaela; Spieß, Katharina 2004: Kinderbetreuung und Fertilität in Deutschland. In: Zeitschrift für Soziologie 33,3: 228-244.

Kalmijn, Matthijs 2005: Attitude alignment in marriage and cohabitation: the case of sex-role attitudes. In: Personal Relationships 12,4: 521-535 [doi: 10.1111/j.1475-6811. 2005.00129.x].

Klein, Thomas; Wunder, Edgar 1996: Regionale Disparitäten und Konfessionswechsel als Ursache konfessioneller Homogamie. In: Kölner Zeitschrift für Soziologie und Sozialpsychologie 48: 96-125.

Kopp, Johannes; Lois, Daniel; Kunz, Christina; Arránz Becker, Oliver 2010: Verliebt, verlobt, verheiratet. Institutionalisierungsprozesse in Partnerschaften. Wiesbaden: VSVerlag.

Kulu, Hill; Milewski, Nadja 2007: Family change and migration in the life course: An introduction. In: Demographic Research 17: 567-590 [doi: 10.4054/DemRes.2007.17.19]

Lankuttis, Teresa; Blossfeld, Hans-Peter 2003: Determinanten der Wiederheirat nach der ersten Scheidung in der Bundesrepublik Deutschland. In: Zeitschrift für Familienforschung 15,1: 5-25.

Lewis, Robert A.; Spanier, Graham B. 1979: Theorizing about the quality and stability of marriage. In: Burr, Wesley (Hrsg.): Contemporary theories about the family (Vol. 1). New York/London: Free Press: 268-294.

Lois, Danie/ 2008: Arbeitsteilung, Berufsorientierung und Partnerschaftsstabilität. Ehen und nichteheliche Lebensgemeinschaften im Vergleich. In: Kölner Zeitschrift für Soziologie und Sozialpsychologie 60: 53-77 [doi: 10.1007/s11577-008-0003-z].

Lois, Danie/ 2011: Church membership and church attendance across time - A trend analysis considering differences between East and West Germany. In: Comparative Population Studies - Zeitschrift für Bevölkerungswissenschaft 36,1: 161-192 [doi: 10.4232/10.CPoS-2011-04en].

Lois, Danie/ 2013: Wenn das Leben religiös macht. Altersabhängige Veränderungen der kirchlichen Religiosität im Lebensverlauf. Wiesbaden: Springer VS.

Milewski, Nadja 2009: Fertility of immigrants: A two-generational approach in Germany. In: Demographic Research Monographs 6. Berlin/Heildelberg: Springer.

Mood, Carina 2010: Logistic Regression: Why we cannot do what we think we can do, and what we can do about it. In: European Sociological Review 26: 67-82 [doi: 10.1093/ esr/jcp006]

Peuckert, Rüdiger 2008: Familienformen im sozialen Wandel. Wiesbaden: VS-Verlag.

Pickel, Gert 2003: Areligiosität, Antireligiosität, Religiosität - Ostdeutschland als Sonderfall niedriger Religiosität im osteuropäischen Rahmen? In: Wohlrab-Sahr, Monika; Pollack, Detlef (Hrsg.): Atheismus und religiöse Differenz. Opladen: Leske + Budrich: 247-270.

Pötzsch, Olga 2012: Geburtenfolge und Geburtenabstand - neue Daten und Befunde. In: Wirtschaft und Statistik 2: 89-101. 
Qian, Zhenchao; Lichter, Daniel T. 2007: Social boundaries and marital assimilation: Interpreting trends in racial and ethnic intermarriage. In: American Sociological Review 72: 68-94 [doi: 10.1177/000312240707200104].

Rös/er, Wiebke 2010: Die ost-westdeutsche Partnerwahl. Wanderungen, Vorurteile, Wohlfahrtsunterschiede. In: Krause, Peter; Ostner, Ilona (Hrsg.): Leben in Ost und Westdeutschland. Eine sozialwissenschaftliche Bilanz der deutschen Einheit 19902010. Frankfurt/M./New York: Campus: 211-226.

Schmollack, Simone 2005: Deutsch-deutsche Beziehungen. Geschichten von der Liebe zwischen Ost und West. Berlin: Schwarzkopf \& Schwarzkopf.

Schneider, Norbert F.; Naderi, Robert; Ruppenthal, Silvia 2012: Familie in Deutschland nach dem gesellschaftlichen Umbruch. Sind Ost-West-Differenzierungen in der Familienforschung zwanzig Jahre nach der Wiedervereinigung noch sinnvoll? In: Huinink, Johannes; Kreyenfeld, Michaela; Trappe, Heike (Hrsg.): Familie und Partnerschaft in West- und Ostdeutschland. Ähnlich und doch immer noch anders. Sonderheft 9 der Zeitschrift für Familienforschung. Berlin/Toronto: Barbara Budrich: 29-54.

Schneider, Norbert F.; Tölke, Angelika; Nauck, Bernhard 1995: Familie im gesellschaftlichen Umbruch - nachholende oder divergierende Modernisierung? In: Nauck, Bernhard; Schneider, Norbert; Tölke, Angelika (Hrsg.): Familie und Lebensverlauf im gesellschaftlichen Umbruch. Stuttgart: Enke: 1-29.

Singer, Judith D.; Willett, John B. 2003: Applied longitudinal data analysis. Modeling change and event occurrence. Oxford: University Press.

Statistisches Bundesamt 2009: Mikrozensus 2008 - Neue Daten zur Kinderlosigkeit in Deutschland. Wiesbaden: Statistisches Bundesamt.

Storch, Kersten 2003: Konfessionslosigkeit in Ostdeutschland. In: Gärtner, Christel; Po/lack, Detlef; Wohlrab-Sahr, Monika (Hrsg.): Atheismus und religiöse Indifferenz. Opladen: Leske + Budrich: 231-245.

Szydlik, Marc; Schupp, Jürgen 2004: Wer erbt mehr? Erbschaften, Sozialstruktur und Alterssicherung. In: Kölner Zeitschrift für Soziologie und Sozialpsychologie 56: 609629 [doi: 10.1007/s11577-004-0106-0].

Trappe, Heike 1995: Emanzipation oder Zwang? Frauen in der DDR zwischen Beruf, Familie und Sozialpolitik. Berlin: Akademie Verlag.

Vatterrott, Anja 2011: The fertility behaviour of East to West German migrants. In: MPIDR Working Paper 2011-13.

Wagner, Gert G.; Frick, J. R.; Schupp, Jürgen 2007: The German Socio-Economic Panel Study (SOEP) - Scope, Evolution and Enhancements. In: Schmollers Jahrbuch 127: 161-191.

Wagner, Michael; Weiß, Bernd 2003: Bilanz der deutschen Scheidungsforschung: Versuch einer Meta-Analyse. In: Zeitschrift für Soziologie 32: 29-49.

Windzio, Mark 2007: Regionale Arbeitslosigkeit und Distanz zur Grenze: Individual- und Kontexteffekte auf die Abwanderung von Arbeitskräften von Ost- nach Westdeutschland. In: Schmollers Jahrbuch 127: 553-583.

Zapf, Wolfgang; Mau, Steffen 1993: Eine demographische Revolution in Ostdeutschland? Dramatischer Rückgang von Geburten, Eheschließungen und Scheidungen. In: Informationsdienst Soziale Indikatoren 10: 1-5. 
Eine Übersetzung dieses begutachteten und vom Autor autorisierten deutschen Originaltextes durch das Bundesinstitut für Bevölkerungsforschung ist unter dem Titel "East-West Couples: Distribution, Characteristics and Stability", DOI 10.12765/CPoS-2014-17en bzw. URN urn:nbn:de:bibcpos-2014-17en9, auf http://www.comparativepopulationstudies.de verfügbar.

Prof. Dr. Daniel Lois $(\varangle)$. Universität der Bundeswehr München, Fakultät für Humanwissenschaften, Department Bildungswissenschaft. München, Deutschland. E-Mail: daniel.lois@unibw.de

URL: http://www.unibw.de/hum/dfb/SWM/pers/daniellois 


\section{Anhang}

Tab. A1: Deskriptive Statistiken zu den Modellvariablen

\begin{tabular}{|c|c|c|c|c|c|}
\hline & $\mathrm{MW} / \%$ & SD & Min & Max & $\%$ imputiert \\
\hline \multicolumn{6}{|l|}{ Paartypen } \\
\hline West-West-Paar, alte BL & $72,1 \%$ & & 0 & 1 & \multirow{4}{*}{15,2} \\
\hline Ost-Ost-Paar, neue BL & $25,7 \%$ & & 0 & 1 & \\
\hline Ost-West-Paar, alte BL & $1,9 \%$ & & 0 & 1 & \\
\hline Ost-West-Paar, neue BL & $0,4 \%$ & & 0 & 1 & \\
\hline \multicolumn{6}{|l|}{ Familiale Lebensformen } \\
\hline NEL ohne Scheidungserfahrung & $7,6 \%$ & & 0 & 1 & \multirow{4}{*}{18,8} \\
\hline NEL mit Scheidungserfahrung & $5,3 \%$ & & 0 & 1 & \\
\hline Ehe ohne Scheidungserfahrung & $75,4 \%$ & & 0 & 1 & \\
\hline Ehe mit Scheidungserfahrung & $11,8 \%$ & & 0 & 1 & \\
\hline Partner haben mindestens ein Kind & $82,0 \%$ & & 0 & 1 & 9,0 \\
\hline Anzahl Kinder 0-1 Jahre im Haushalt & 0,04 & 0.2 & 0 & 3 & \\
\hline Anzahl Kinder 2-4 Jahre im Haushalt & 0,1 & 0.3 & 0 & 3 & 0,0 \\
\hline Anzahl Kinder über 4 Jahre & 0,2 & 0.5 & 0 & 4 & \\
\hline \multicolumn{6}{|l|}{ Ökonomische Lage und Arbeitsteilung } \\
\hline Index ökonomische Lage ${ }^{\#}$ & 0,0 & 1.0 & -3.3 & 11.2 & 8,8 \\
\hline Ein Partner arbeitslos & $20,4 \%$ & & 0 & 1 & \multirow{3}{*}{12,6} \\
\hline Beide Partner arbeitslos & $6,6 \%$ & & 0 & 1 & \\
\hline Erwerbsindex $\#$ & 6,4 & 4.1 & 0 & 12 & \\
\hline Erwerbsindex (Mann-Frau) & 3,2 & 5.8 & -12 & 12 & \multirow{3}{*}{19,7} \\
\hline Hausarbeitsstunden Wochenende & 1,8 & 0.9 & 0 & 10 & \\
\hline Hausarbeit (Mann-Frau) & $-1,9$ & 1.8 & -10 & 10 & \\
\hline \multicolumn{6}{|l|}{ Kirchliche Religiosität } \\
\hline Kirchgangshäufigkeit $\#$ & 9,2 & 13.6 & 0 & 52 & \multirow{2}{*}{18,8} \\
\hline Kirchgang (|Mann-Frau|) & 5,0 & 10.1 & 0 & 52 & \\
\hline Beide Partner konfessionslos & $20,2 \%$ & & 0 & 1 & \\
\hline Konfessionelle Heterogamie & $26,8 \%$ & & 0 & 1 & 31,4 \\
\hline Konfessionelle Homogamie & $53,1 \%$ & & 0 & 1 & \\
\hline \multicolumn{6}{|l|}{ Zeitindikatoren } \\
\hline Alter $\#$ & 47,5 & 14.4 & 17 & 95 & 1,0 \\
\hline Ehedauer (codiert mit 0 bei NEL, +1) & 20,4 & 15.6 & 1 & 72 & 22,0 \\
\hline Zeitraum 1991-1995 & $19,4 \%$ & & 0 & 1 & \\
\hline Zeitraum 1996-2000 & $23,2 \%$ & & 0 & 1 & \\
\hline Zeitraum 2001-2005 & $33,6 \%$ & & 0 & 1 & 0,0 \\
\hline Zeitraum 2006-2009 & $23,8 \%$ & & 0 & 1 & \\
\hline Kohorte ab 1971 (Ref.: bis 1970) & $14,3 \%$ & & 0 & 1 & \\
\hline Wohnort Berlin & $3,5 \%$ & & 0 & 1 & 7,1 \\
\hline n (Paare) & & & 16.372 & & \\
\hline n (Beobachtungen) & & & 122.049 & & \\
\hline
\end{tabular}

$\mathrm{BL}=$ Bundesländer, NEL $=$ Nichteheliche Lebensgemeinschaft, $\mathrm{SD}=$ Standardabweichung MW = Mittel- und Anteilswerte; beziehen sich auf die 122.049 Beobachtungen im Long-Format

\# = operationalisiert als Paarmittelwert

Quelle: SOEP (1990-2009, eigene Berechnungen) 


\section{Comparative Population Studies}

WWW.comparativepopulationstudies.de

ISSN: 1869-8980 (Print) - 1869-8999 (Internet)

Published by / Herausgegeben von

Prof. Dr. Norbert F. Schneider

Federal Institute for Population Research

D-65180 Wiesbaden / Germany

\section{Managing Editor /}

Verantwortlicher Redakteur

Frank Swiaczny

\section{Assistant Managing Editor /}

\section{Stellvertretende Redakteurin}

Katrin Schiefer

\section{Copy Editor (German) /}

Lektorat (deutsch)

Dr. Evelyn Grünheid

\section{Layout / Satz}

Beatriz Feiler-Fuchs

E-mail:cpos@bib.bund.de

\section{Scientific Advisory Board /}

Wissenschaftlicher Beirat

Paul Gans (Mannheim)

Johannes Huinink (Bremen)

Michaela Kreyenfeld (Rostock)

Marc Luy (Wien)

Clara H. Mulder (Groningen)

Notburga Ott (Bochum)

Peter Preisendörfer (Mainz)

Zsolt Spéder (Budapest)
Board of Reviewers / Gutachterbeirat Martin Abraham (Erlangen)

Laura Bernardi (Lausanne)

Hansjörg Bucher (Bonn)

Claudia Diehl (Konstanz)

Andreas Diekmann (Zürich)

Gabriele Doblhammer-Reiter (Rostock)

Jürgen Dorbritz (Wiesbaden)

Anette Eva Fasang (Berlin)

E.-Jürgen Flöthmann (Bielefeld)

Alexia Fürnkranz-Prskawetz (Wien)

Beat Fux (Salzburg)

Joshua Goldstein (Berkeley)

Karsten Hank (Köln)

Sonja Haug (Regensburg)

Hill Kulu (Liverpool)

Aart C. Liefbroer (Den Haag)

Kurt Lüscher (Konstanz)

Emma Lundholm (Umeå)

Nadja Milewski (Rostock)

Dimiter Philipov (Wien)

Roland Rau (Rostock)

Tomáš Sobotka (Wien)

Jeroen Spijker (Barcelona)

Olivier Thévenon (Paris)

Helga de Valk (Brussel)

Heike Trappe (Rostock)

Michael Wagner (Köln) 